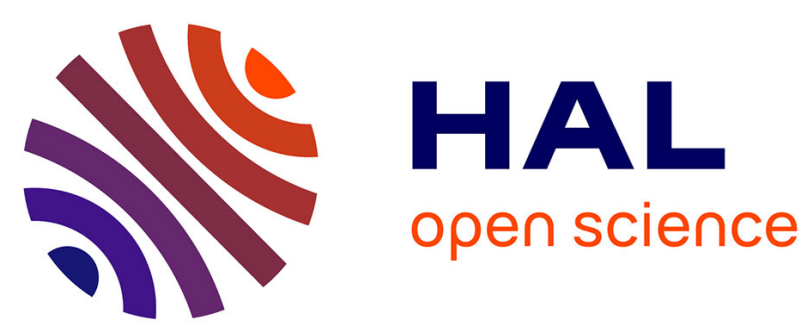

\title{
L'information Géographique
}

Ana Roxin

\section{To cite this version:}

Ana Roxin. L'information Géographique. Géopositionnement et mobilités (GPS, Egnos et Galileo), PRUR-UTBM, pp.57-91, 2009, 978-2-914279-40-6. hal-00877057

\section{HAL Id: hal-00877057 https://hal.science/hal-00877057}

Submitted on 28 Oct 2021

HAL is a multi-disciplinary open access archive for the deposit and dissemination of scientific research documents, whether they are published or not. The documents may come from teaching and research institutions in France or abroad, or from public or private research centers.
L'archive ouverte pluridisciplinaire HAL, est destinée au dépôt et à la diffusion de documents scientifiques de niveau recherche, publiés ou non, émanant des établissements d'enseignement et de recherche français ou étrangers, des laboratoires publics ou privés. 


\section{L'information géographique}

Ana ROXIN

UTBM, laboratoire SeT, équipe de recherche GSEM (Géopositionnement, Systèmes Embarqués et Mobilité)

\section{Introduction}

Dès l'Antiquité, les hommes ont cherché à représenter leur territoire et à mémoriser des itinéraires.

Au fil du temps, divers instruments sont apparus, permettant de mesurer les angles, de déterminer latitude et longitude, ou encore de mesurer les altitudes avec précision. Des systèmes de projection, comme celui de Mercator, ont permis la représentation de l'ensemble du globe en deux dimensions. C'est grâce à ces méthodes que la cartographie a commencé à présenter un intérêt grandissant, au début surtout pour des raisons militaires. En effet, ce sont les deux grandes Guerres Mondiales qui ont stimulé la production de cartes à grande échelle, d'abord en Europe, puis sur les autres continents.

De nos jours, la cartographie connaît une véritable révolution de part la création de données numériques et de leur diffusion sur I'Internet. Le développement des capteurs numériques (radar et laser) et le déploiement de satellites d'observation de la Terre, permet aujourd'hui de décrire des zones terrestres inaccessibles auparavant, telles l'Antarctique.

Aujourd'hui, les informations géographiques proviennent de sources variées, et sont de différents types. Cette diversité amène le problème de la gestion de la qualité de ces informations géographiques, autrement dit de leur précision, degré de mise à jour, exhaustivité et cohérence logique.

Dans ce chapitre, nous allons étudier comment sont collectées les données géographiques, ainsi que les différents types de représentation de l'information géographique.

\section{Information Géographique (IG) : présentation}

\section{Définition de l'Information Géographique}

Une information géographique est souvent définie en tant qu'une description d'un objet réel ayant une dimension spatiale, tel une rivière, un village ou une région [1]. Cette description se traduit par une schématisation de la dimension, la forme, le comportement et la localisation de l'objet.

L'information géographique doit permettre d'étudier la réalité d'un territoire avec les objets qui le compose. Or pour ce faire, il faut d'abord schématiser cette réalité, ce qui est assez difficile, à réaliser notamment à cause de la multitude d'objets complexes, de leurs descriptions, des relations entre ces divers objets, etc.

En conséquence, étant donnée la complexité du monde réel, celui-ci ne peut être modélisé qu'en fonction d'un objectif donné, car il serait impossible de le modéliser de manière universelle. L'information géographique n'existe donc pas en tant que telle. Elle existe toujours par rapport à un point de vue et un objectif donné. La description d'un objet géographique étant différente selon l'objectif visé par l'observateur, plusieurs informations géographiques peuvent faire référence à un même objet géographique.

Il est dès lors important de différencier les données contenues dans une information géographique. Généralement, deux types de données sont définis [1]:

- Les données sémantiques (ou les attributs) - données constituant une description nonlocalisée de l'objet géographique ; 
- Les données géométriques ou l'attribut de localisation - données décrivant la localisation de l'objet géographique, en général ce sont des coordonnées dans un système de référence géodésique ou de projection cartographique.

Cette différenciation est importante car c'est l'attribut de localisation qui détermine la position spatiale des données sémantiques. Par exemple, si l'on choisit de modéliser une rivière, l'information géographique associée comportera les données suivantes :

- Données sémantiques - la largeur de son lit, sa longueur, la vitesse du courant, son débit, ses affluents, etc. ;

- Données géométriques - sa localisation et sa topologie (les relations et la connectique entre les éléments constituant la rivière).

On voit ici que les attributs tels les affluents ou la largeur de la rivière sont intimement liés à la localisation géographique de la rivière.

\section{Représentations de l'Information Géographique}

Les différents types de données compris dans une information géographique donnent naissance à différentes représentations de cette information géographique.

\section{Représentation maillée}

Le mode maillé permet de représenter une zone de l'espace à travers une grille de pixels de taille régulière. Chaque pixel (ou cellule de grille) couvre une aire géographique donnée et une valeur d'attribut est assignée à la cellule. La cellule de la grille est la plus petite unité géographique dans une représentation maillée.

La résolution dépend de la taille de la cellule de la grille. Plus la cellule est grande, moins l'information est précise et plus la cellule est petite, plus la résolution est grande [2].

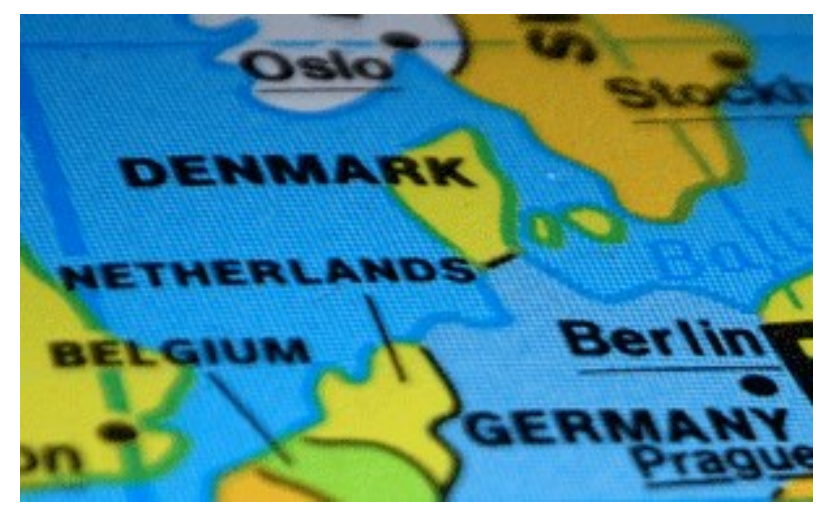

Figure 1. Exemple d'une représentation maillée.

\section{Représentation vectorielle}

La représentation vectorielle permet d'afficher les données géographiques sous forme de points, lignes, courbes, ou surfaces, avec des attributs. Ce type de représentation permet de facilement représenter les formes linéaires, de type surface ou complexes.

Chaque objet géographique est représenté par plusieurs points, reliés entre eux par des segments de droites ou par des courbes [3]. Tous les points sont localisés à travers leurs coordonnées.

La représentation des points est immédiate, puisqu'ils sont décrits par leurs coordonnées dans un système de référence donné. 


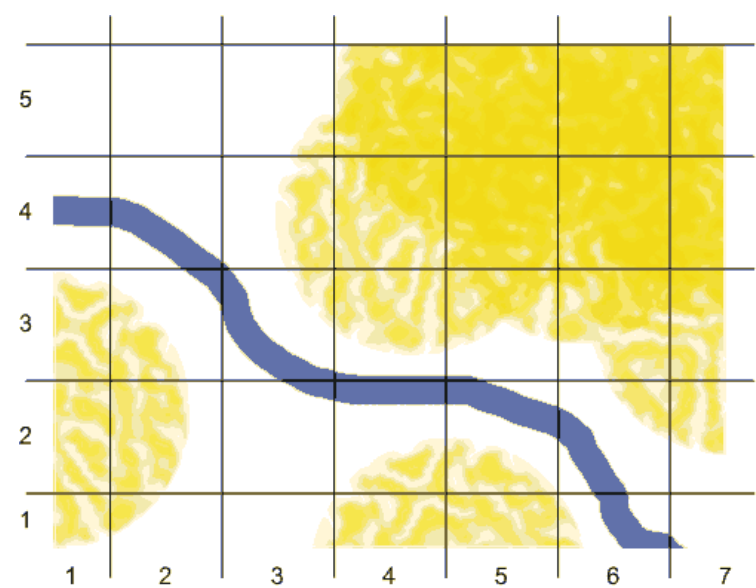

Image raster d'une rivière et des montagnes

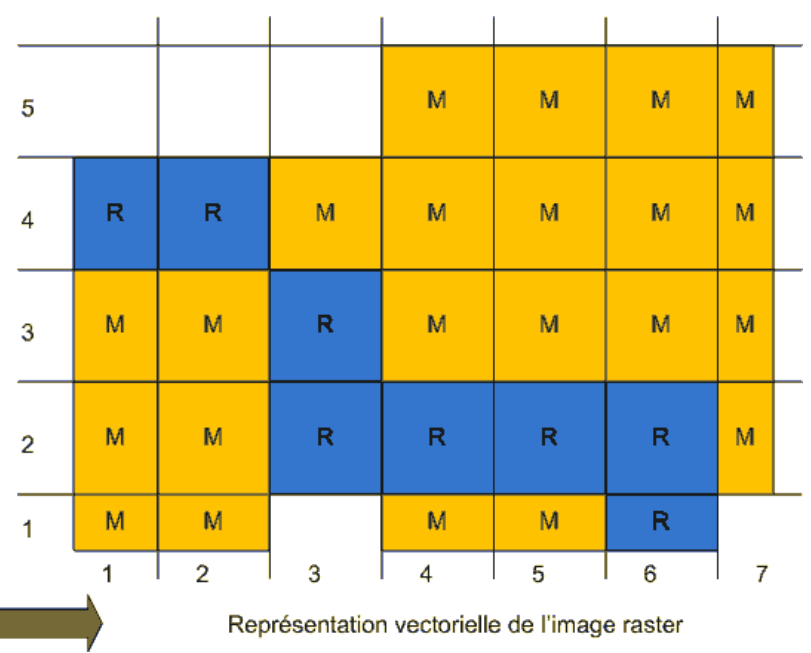

Figure 2. Comparaison entre une représentation maillée (à gauche) et une représentation vectorielle (à droite).

\section{Exemples de représentations de l'Information Géographique}

Trois principales formes de représentation seront présentées ci-dessous :

- $\quad$ La carte ;

- L'image numérique ;

- Le « web mapping ».

\section{La carte}

Au fil des siècles, la carte a évolué d'une projection de la représentation que l'homme se faisait du monde, vers un véritable outil de décision de nos jours. Les cartes sont principalement utilisées pour l'aménagement du territoire, le voyage, la navigation, la géopolitique, ou tout simplement pour diffuser la connaissance [5].

Le Comité Français de Cartographie définit une carte en tant qu'une « représentation géométrique conventionnelle, généralement plane, en positions relatives, de phénomènes concrets ou abstraits, localisables dans l'espace » [5]. Généralement, une carte est une représentation graphique, réalisée en deux dimensions, à une échelle donnée, d'objets géographiques (naturels ou construits par l'Homme, sur ou sous la surface de la Terre ou d'autres planètes).

Sur une carte, les caractéristiques des objets géographiques sont placées le plus précisément possible, en respectant un système de coordonnées [4]. Une carte représente à la fois des données sémantiques et des données géométriques. Les données sémantiques sont généralement représentées à travers des symboles, dont les attributs sont expliqués par la légende de la carte.

La représentation de ces données se fait en respectant un rapport de similitude prédéfini, appelé échelle. L'échelle d'une carte est définie comme le rapport entre la distance mesurée sur la carte et la distance réelle sur le terrain [5]. Les différents rapports d'échelle correspondent chacun à un groupe de domaines d'applications (voir la Figure 3) [6]. 


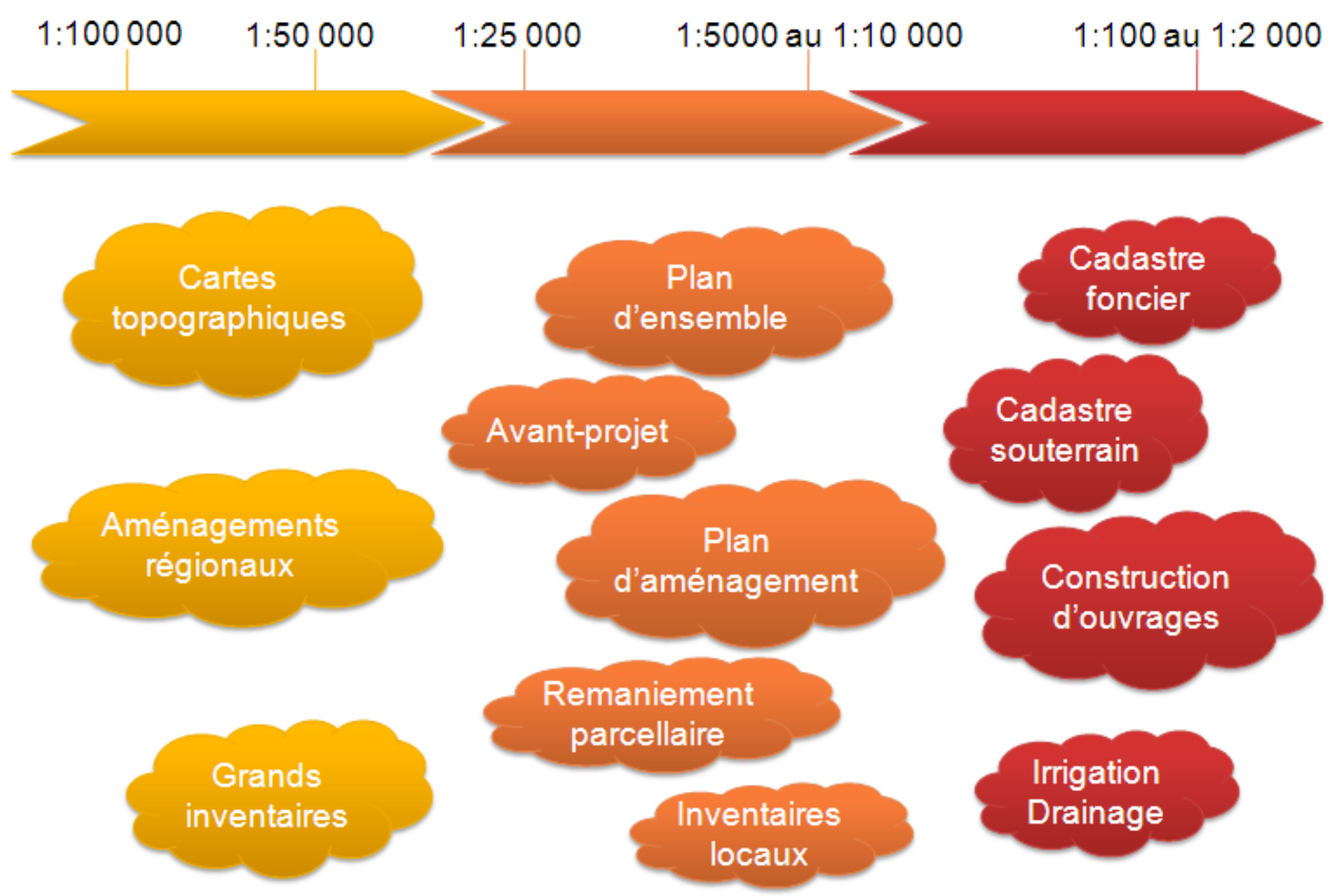

Figure 3. Echelles de cartes et domaines d'application associés [6].

Notamment, dans le domaine du transport, ce sont les cartes topographiques, les cartes routières ou encore les plans (de ville, de réseaux de transports publics, etc.) qui sont les plus utilisées. La carte topographique est une «carte de base comprenant les éléments essentiels à la localisation » [5], notamment les routes, le relief, etc. Un exemple d'une telle carte est donné par la Figure 4. La Figure 5 illustre une carte topographique routière.

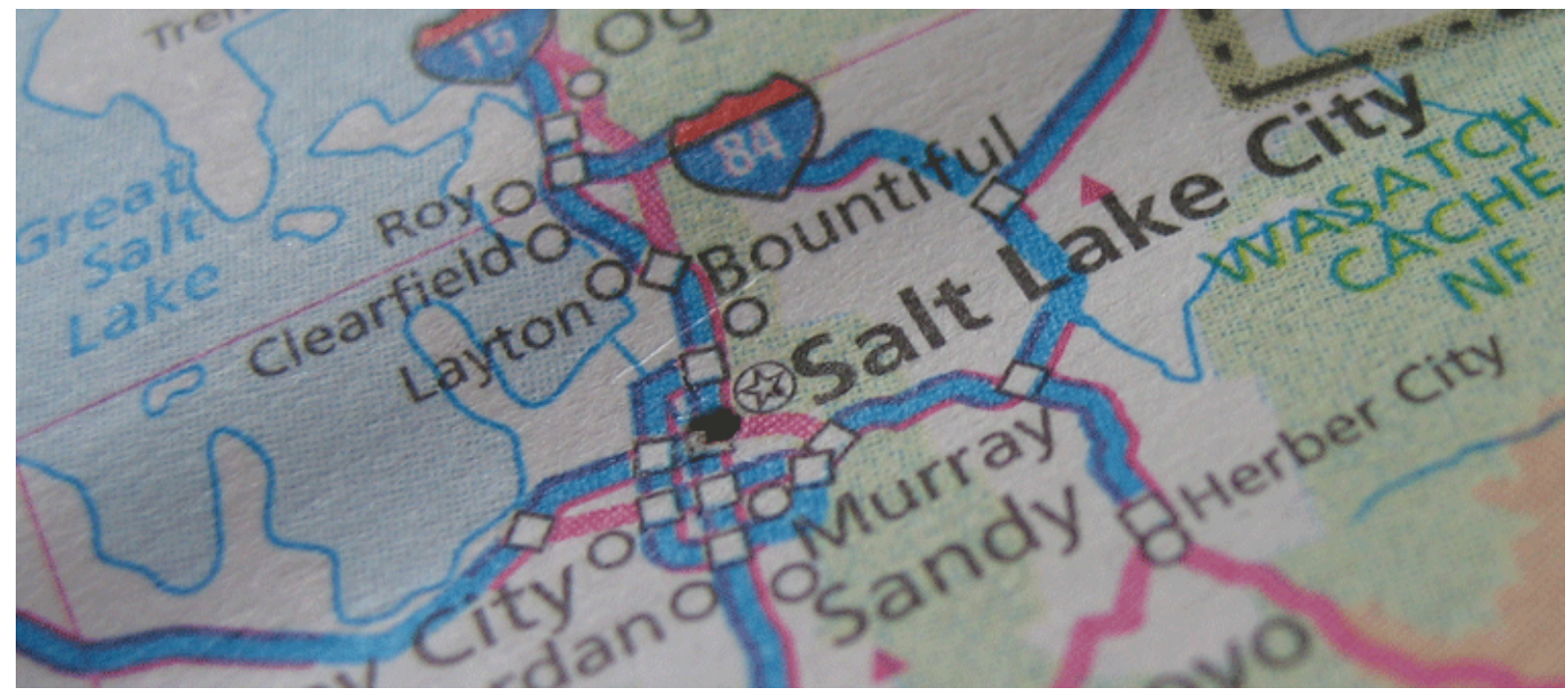

Figure 4. Exemple de carte topographique. 


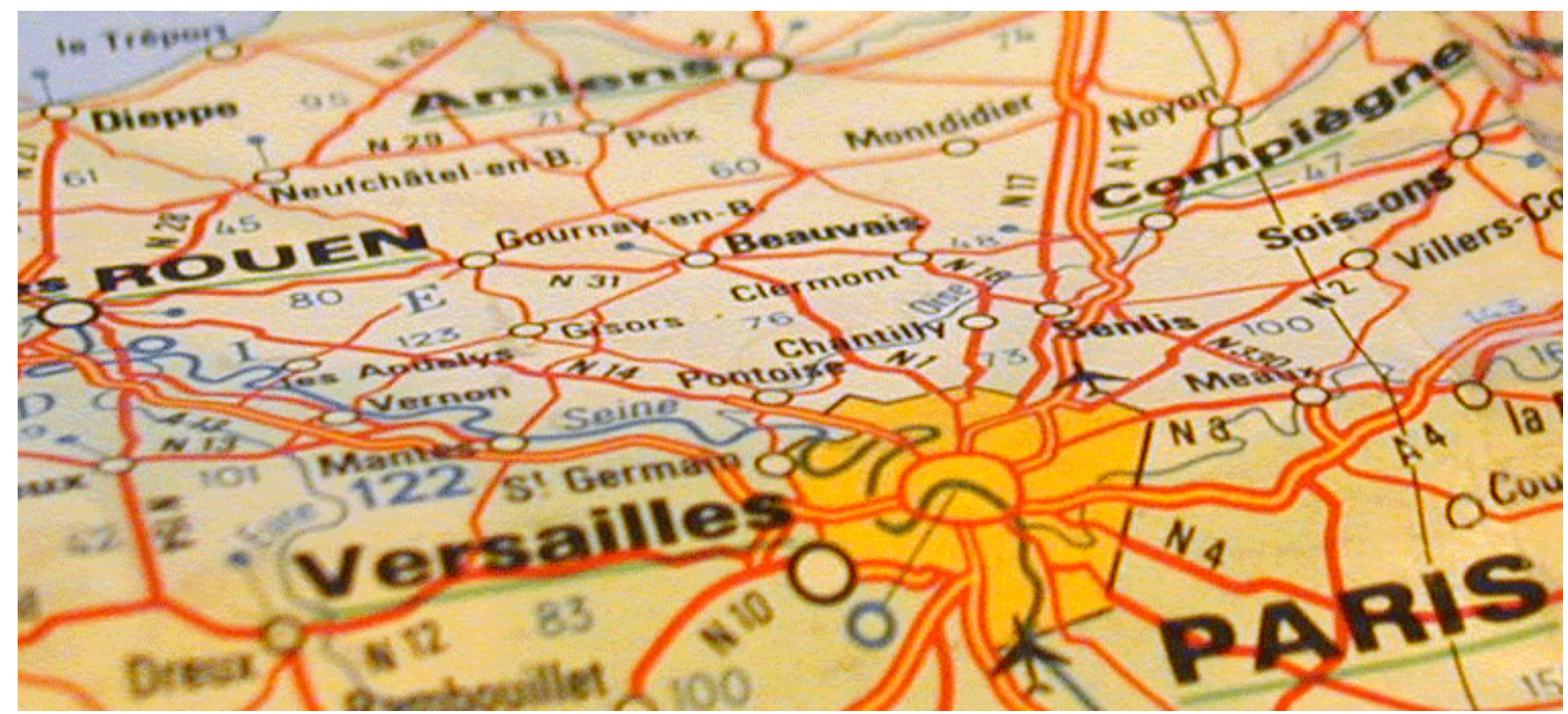

Figure 5. Exemple de carte routière topographique.

\section{L'imagerie numérique}

De nos jours, l'information géographique se retrouve de plus en plus sous forme d'images numériques enregistrées. Le terme « image numérique » correspond à une image enregistrée et géocodée d'une portion de la surface de la Terre. Il s'agit notamment d'images satellitaires ou d'ortho-photographies (voir la section "Sources de référence").

\section{Le "web mapping»}

Le « web mapping » est le processus de création, implémentation, génération et distribution de cartes à travers Internet. Le «web mapping» est la branche qui traite surtout les problèmes d'ordre technologique posés par une telle publication des données géographiques et thématiques. Cette discipline étudie l'usage des cartes sur Internet, l'évaluation et l'optimisation des techniques et processus, ou encore des aspects sociaux liés à ces usages.

Concernant les différentes catégories de cartes Internet, de nombreux efforts de classification ont été réalisés [9-11]. Cependant, ces classifications sont loin d'être encore d'actualité aujourd'hui, au vu du nombre grandissant de possibilités offertes par le « web mapping».

Le Tableau 1 présente une liste des principaux types de cartes Internet présentes sur la Toile aujourd'hui.

\begin{tabular}{|l|l|}
\hline Type carte & Description \\
\hline Cartes Internet statiques & $\begin{array}{l}\text { Ce sont des cartes qui ne permettent que l'affichage (pas } \\
\text { d'interactivité, pas d'animation). Ces cartes sont créées une seule } \\
\text { fois, et sont rarement mises à jour. Les principaux formats sont le } \\
\text { JPEG, GIF ou PNG, pour les cartes raster, (voir 2.2.1.) ou encore le } \\
\text { SVG ou PDF, pour les cartes vectorielles (voir 2.2.2.). Souvent il } \\
\text { s'agit de cartes papier qui ont été numérisées et dont le rendu final } \\
\text { sur un écran d'ordinateur est bien inférieur à leur rendu initial, sur } \\
\text { papier. }\end{array}$ \\
\hline Cartes Internet dynamiques & $\begin{array}{l}\text { Ces cartes sont générées à la demande de l'utilisateur, à chaque } \\
\text { fois que celui- ci actualise la page Internet. Ces cartes sont créées } \\
\text { à partir de bases de données géographiques. }\end{array}$ \\
\hline
\end{tabular}




\begin{tabular}{|c|c|}
\hline Cartes Internet distribuées & $\begin{array}{l}\text { Ces cartes sont créées à partir de sources de données distribuées. } \\
\text { C'est principalement à travers de protocoles tels le protocole WMS } \\
\text { (Web Map Server), défini par l'OGC (Open Geospatial Consortium), } \\
\text { que se fait l'accès à des cartes situées sur un serveur distant. Un } \\
\text { serveur WMS permet, par exemple, de collecter des couches } \\
\text { topographiques puis d'y superposer des couches de données } \\
\text { thématiques. }\end{array}$ \\
\hline Cartes Internet animées & $\begin{array}{l}\text { Ce type de cartes permet de représenter des changements } \\
\text { survenus au cours du temps, en animant une variable graphique ou } \\
\text { temporelle. Les formats de fichiers correspondant à ce type de } \\
\text { cartes sont le SVG (voir } 3.2 \text { ), Flash, Java ou encore QuickTime. Un } \\
\text { exemple d'une telle carte est disponible à partir de l'adresse } \\
\text { suivante: } \\
\text { http://mappemonde.mgm.fr/num14/articles/samu ani.html }\end{array}$ \\
\hline Cartes Internet temps-réel & $\begin{array}{l}\text { Ces cartes permettent d'observer des phénomènes donnés en quasi } \\
\text { temps-réel. Les données sont généralement collectées à partir de } \\
\text { capteurs, envoyés à travers Internet vers un serveur central, puis } \\
\text { les cartes sont générées ou mises à jour à des intervalles réguliers. } \\
\text { Un des exemples les plus éloquents est constitué par les cartes de } \\
\text { trafic ou encore par les systèmes de gestion de flottes de véhicules. } \\
\text { La Figure } 6 \text { illustre une carte de ce type. }\end{array}$ \\
\hline Cartes Internet personnalisées & $\begin{array}{l}\text { Pour ce type de cartes, l'utilisateur peut choisir lui-même les } \\
\text { couches à afficher. Ces cartes permettent un filtrage des données, } \\
\text { un contenu sélectif et un affichage personnalisé. }\end{array}$ \\
\hline Cartes Internet réutilisables & $\begin{array}{l}\text { Sous ce nom, on retrouve des systèmes plus complexes qui } \\
\text { proposent des API (Application Programming Interface) pouvant } \\
\text { être réutilisées sur d'autres sites Internet ou pour d'autres produits. }\end{array}$ \\
\hline Cartes Internet interactives & $\begin{array}{l}\text { Ce type de carte permet d'explorer des cartes, en modifier certains } \\
\text { paramètres, naviguer et interagir avec la carte. D'un point de vue } \\
\text { technique, ceci est réalisé à travers une combinaison d'événements, } \\
\text { scripts et DOM (Document Object Model). }\end{array}$ \\
\hline Cartes Internet analytiques & $\begin{array}{l}\text { Ces cartes permettent d'avoir accès à une analyse de type SIG } \\
\text { (Système d'Informations Géographiques), les géodonnées étant } \\
\text { soit fournies soit chargées par l'utilisateur. }\end{array}$ \\
\hline Cartes Internet collaboratives & $\begin{array}{l}\text { Ces cartes sont assez récentes et assez complexes à implémenter. } \\
\text { L'idée est d'avoir plusieurs utilisateurs à différents endroits du Globe } \\
\text { pouvant collaborer pour créer et améliorer des cartes à travers } \\
\text { Internet. Dans cette catégorie, on peut citer les projets } \\
\text { OpenStreetMap (http://www.openstreetmap.org/) ou WikiMapia } \\
\text { (http://wikimapia.org/). }\end{array}$ \\
\hline
\end{tabular}

Tableau 1. Types de cartes Internet. 
Mise à jour 21/11/2008 09:30
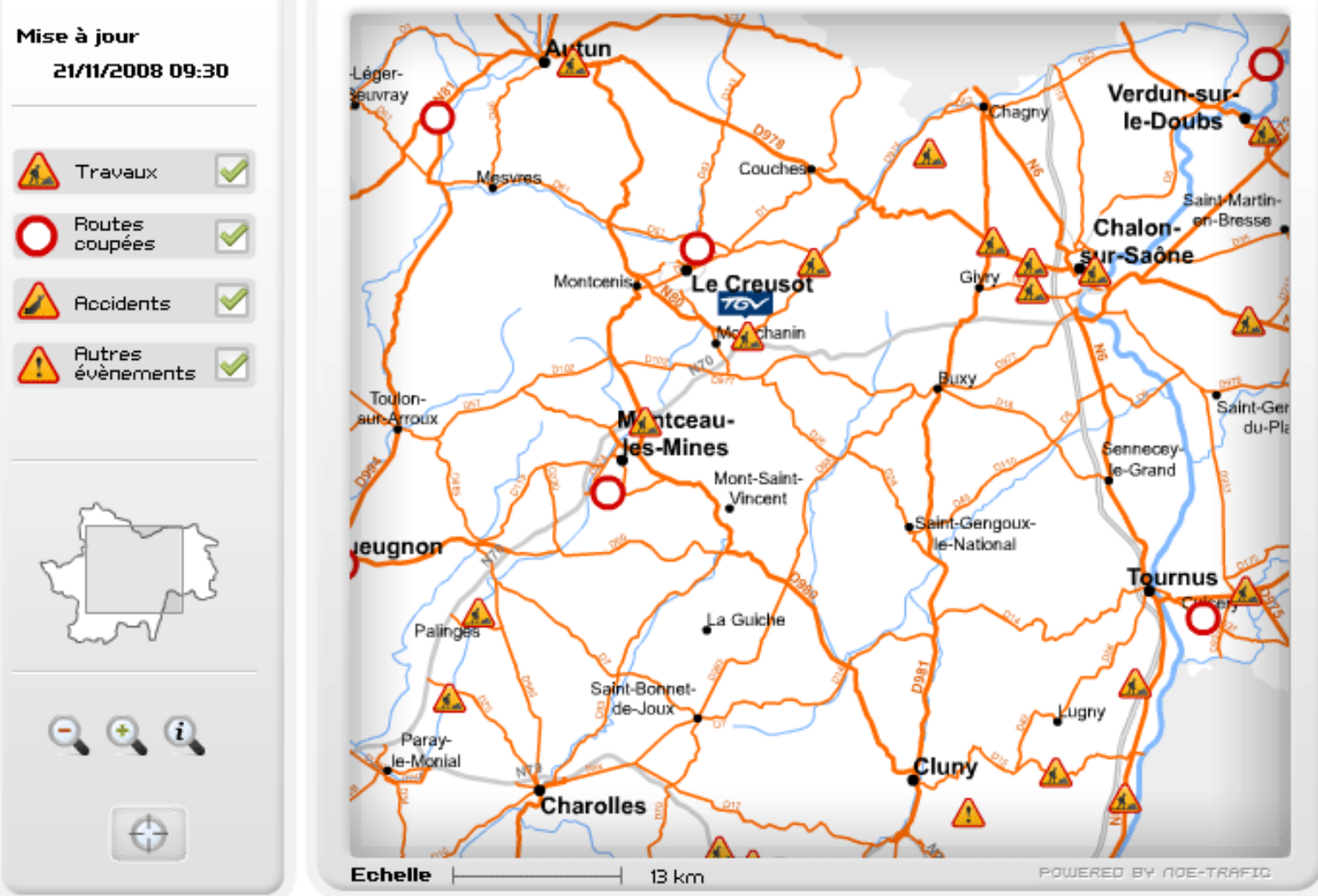

Figure 6. Exemple d'une carte Internet temps-réel, telle qu'affichée sur le site du Conseil Général 71 (http://www.cg71.fr/jahia/Jahia/pid/25) 


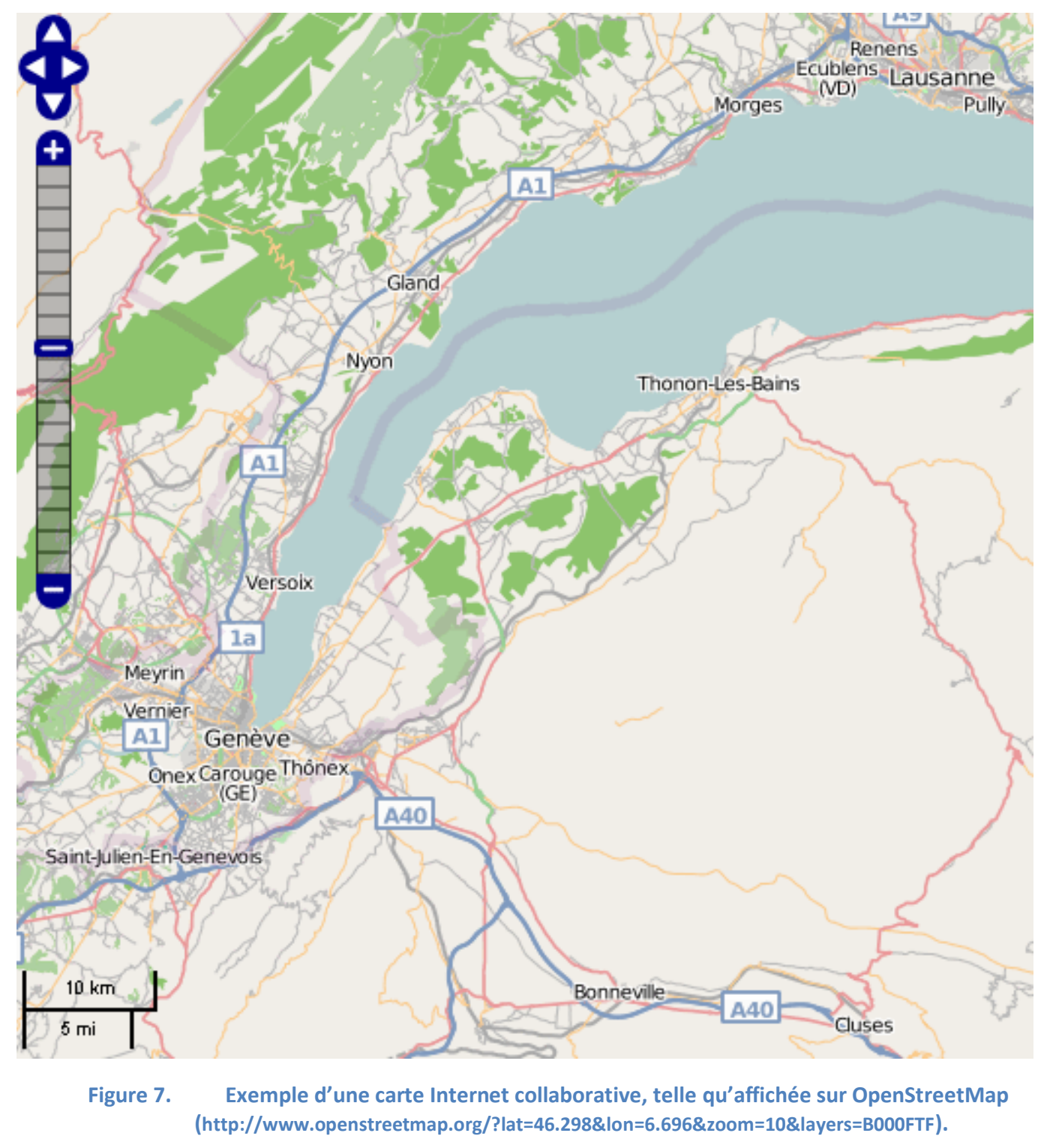

Un autre exemple de «web mapping» est offert par portail mis en place dans le cadre du projet européen INSPIRE (Infrastructure for Spatial Information in Europe). Ce portail est accessible en ligne depuis l'adresse suivante : http://www.inspire-geoportal.eu/maps.htm. Les différentes fonctionnalités offertes permettent de créer des cartes à partir de différents serveurs cartographiques répartis dans le monde, gérer les couches d'une carte ainsi créée, mais aussi de sauvegarder ces cartes. Les données disponibles couvrent l'Europe entière. La Figure 8 est un exemple d'une carte générée sur ce portail. La carte est créée à partir d'une fond cartographique (en occurrence l'Europe), puis des informations sont chargées à partir d'un serveur WMS (en occurrence des informations concernant l'érosion des sols, indiquée par des zones rectangulaires).

Toujours dans le cadre du projet INSPIRE, le BRGM (Bureau de Recherches Géologiques et Minières) a mis en ligne le système InfoTerre (http://infoterre.brgm.fr/). InfoTerre permet aux utilisateurs d'avoir accès à différentes données géographiques provenant de plusieurs organismes différents : le BRGM (Bureau de Recherches Géologiques et Minières), I'IGN (Institut Géographique Nationa), I'IFEN (Institut Français de /'Environnement) et Cartosphère (http://www.cartosphere.com/). L'utilisateur peut sélectionner les couches cartographiques qu'il souhaite visualiser. Les données sont seulement disponibles pour la France: 
- BRGM - Cartes géologiques, dossiers sur le sous-sol, eaux, risques naturels, sites et sols pollués, géophysique, inventaire minier, ou encore géologie marine ;

- Cartosphère - Fond cartographique constitué d'images raster en provenance de TeleAtlas à des échelles allant du 1:4 000 à 1:4 000000 ;

- $\quad$ IFEN - Occupation des sols ;

- IGN - BD CARTHAGE (réseau hydrographique), BD CARTO (limites administratives, départements, régions).

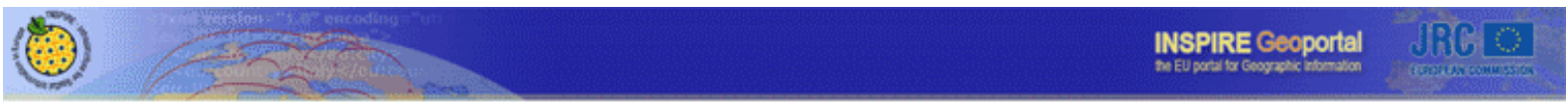

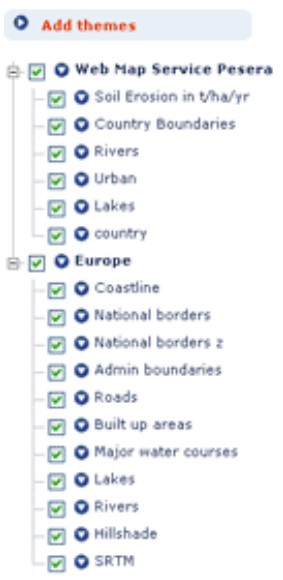

Q Reload the current map

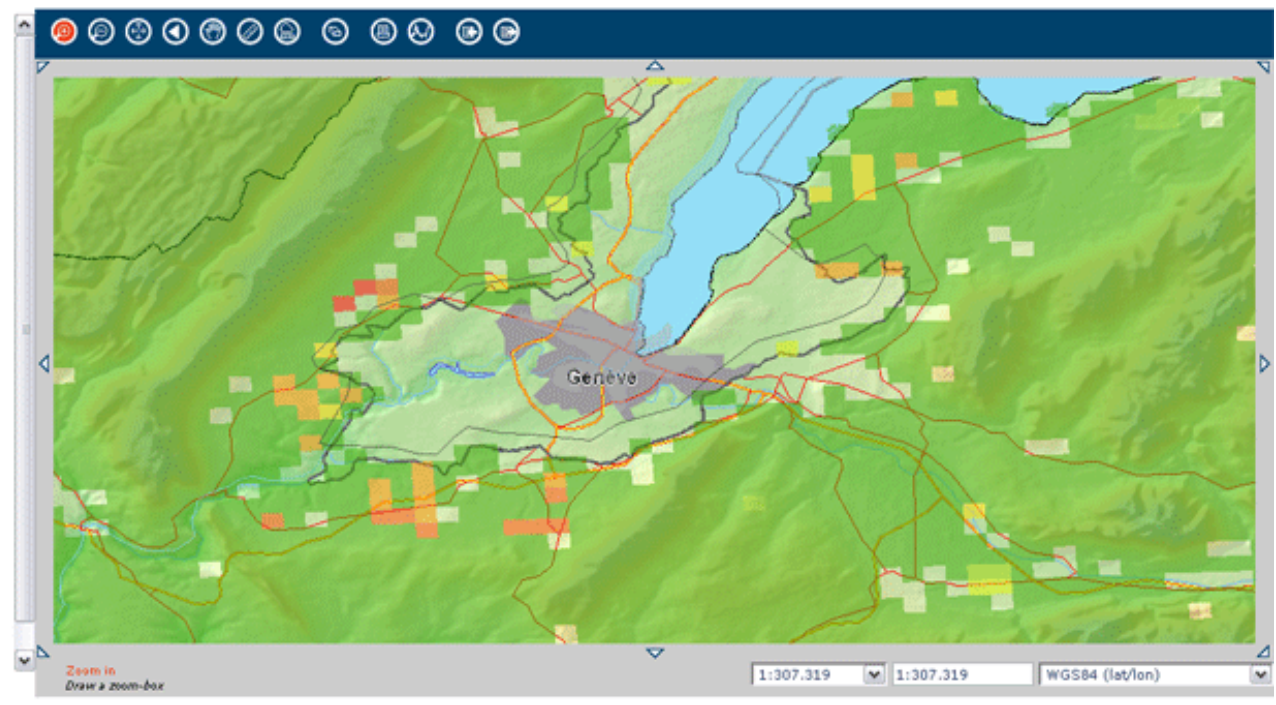

Figure 8. Exemple d'une carte générée à partir du portail INSPIRE.

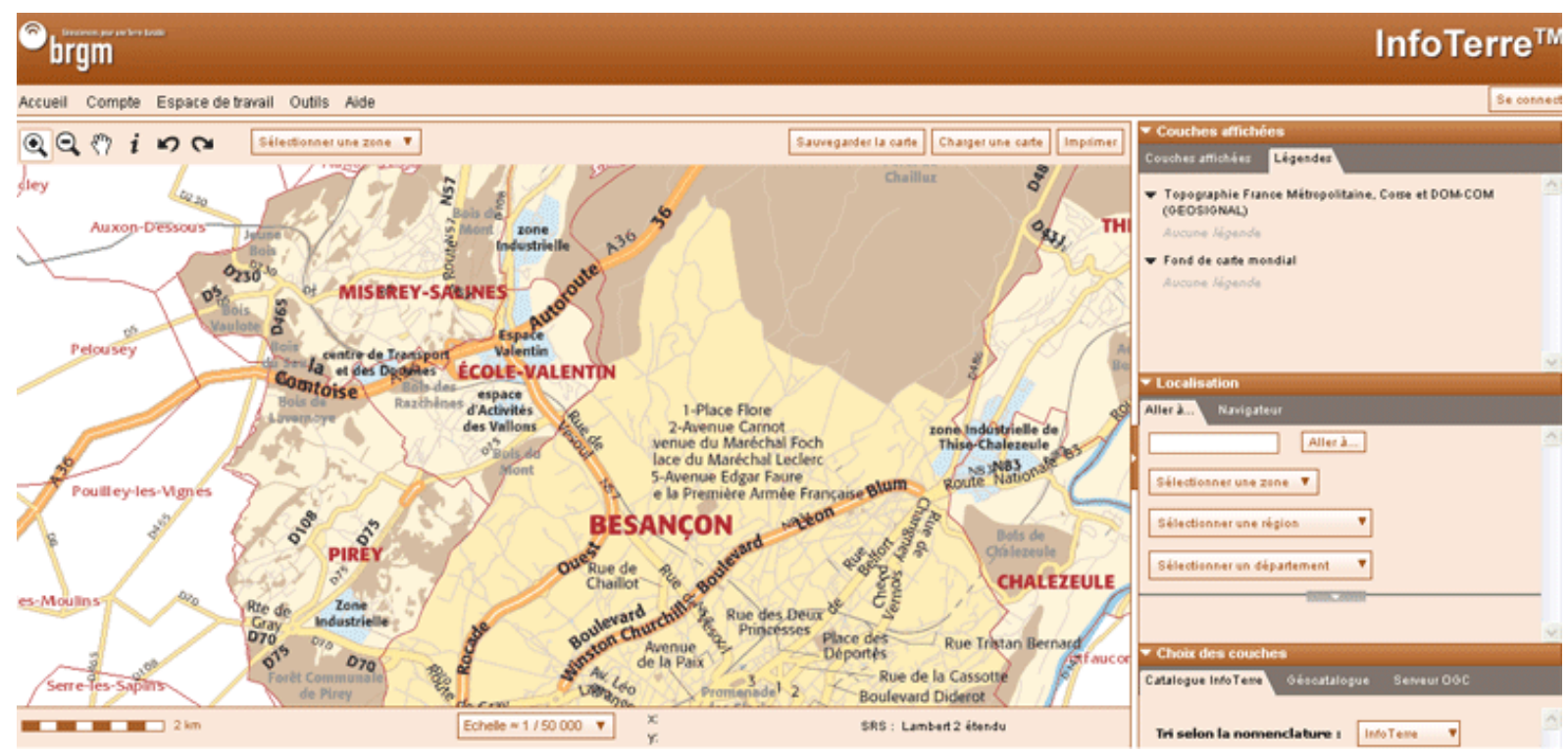

Figure 9. Exemple d'une carte générée à partir du portail InfoTerre ${ }^{\mathrm{TM}}$.

Parmi les principaux avantages du « web mapping », on peut citer les suivants: 
- Le «web mapping» permet de distribuer des informations géographiques actualisées régulièrement. Si les cartes web sont générées à partir de bases de données géographiques, elles peuvent afficher des informations en quasi temps-réel. Un exemple d'un tel usage serait une carte affichant l'état du trafic dans le voisinage de l'utilisateur.

- $\quad$ Si elles sont implémentées correctement, les cartes Internet peuvent être affichées quelque soit le système d'exploitation de l'utilisateur ou encore son navigateur Internet. De plus, il est très aisé d'intégrer des données multimédia aux données géographiques contenues dans les cartes Internet. Les cartes Internet deviennent un important vecteur d'information, permettant entre autres, l'encapsulation de liens Internet vers d'autres sources d'information. Par exemple, la carte d'une ville peut contenir des liens vers les horaires pour chaque arrêt de bus dans la ville.

- $\quad$ Le « web mapping » permet aussi d'accéder à une « cartographie collaborative ». A la manière du projet Wikipedia, il existe des technologies pour le « web mapping » (Java, Flash, SVG, DHTML/Ajax) permettant l'acquisition de données provenant de sources distribuées ou encore une collaboration dans la création de cartes Internet. A ce titre, on peut citer le projet OpenStreetMap (http://www.openstreetmap.org/) ou encore la communauté WikiMapia (http://wikimapia.org/).

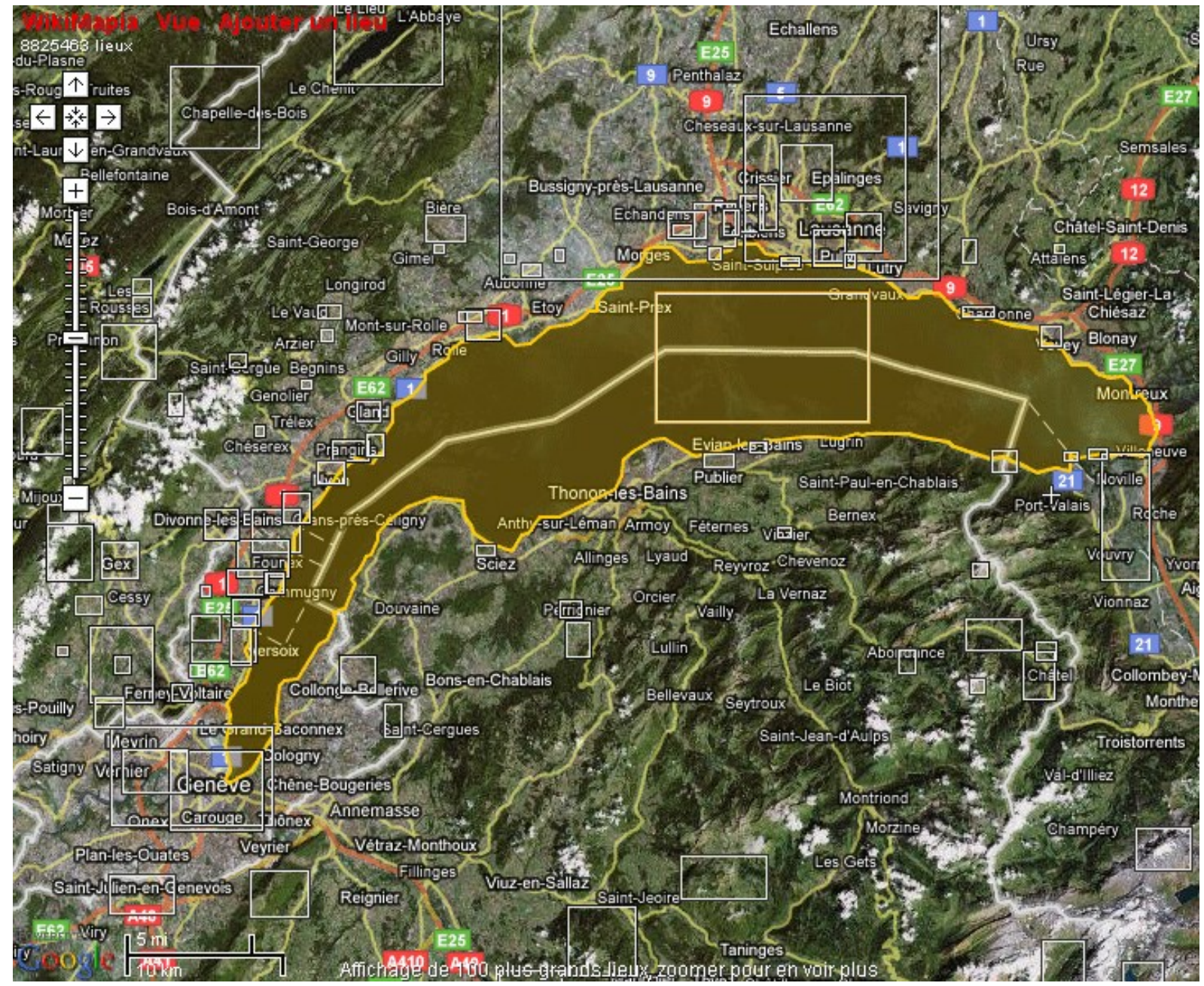

Figure 10. Exemple de carte créée par la communauté WikiMapia. En cliquant sur le Lac Léman, on accède à des informations le concernant (telles ses dimensions ou sa position géographique), et ce dans une vingtaine de langues. L'utilisateur peut modifier ou ajouter un commentaire. Chaque zone rectangulaire sur la carte repère des objets géographiques pour lesquels les utilisateurs de la communauté ont défini des commentaires.

Cependant, il existe aussi des inconvénients liés au « web mapping»:

- $\quad$ La fiabilité de l'Internet et des infrastructures type serveur Web n'est pas encore parfaite. Si une carte fait référence à des sources de données distribuées (notamment sur des serveurs WMS), sa disponibilité ne peut pas être garantie. En effet, à tout moment, il est possible que des données distantes 
soient inaccessibles. Lorsque l'utilisateur voudra afficher la carte contenant ces données, des erreurs seront générées.

- $\quad$ En dépit de la quantité grandissante d'outils pour la création de cartes Internet, le procédé de création de cartes Internet demeure fastidieux. L'utilisateur doit connaître, intégrer et maîtriser des technologies, services et sources de données variées. A titre d'exemple, on peut citer le projet Open $\operatorname{Map}^{\mathrm{TM}}$ (http://openmap.bbn.com/demo/), qui utilise la technologie Java afin de permettre aux utilisateurs de créer des cartes en partant d'un fond cartographique donné (généralement une carte mondiale incluant les frontières des pays). La Figure 11 illustre la complexité d'une telle approche.

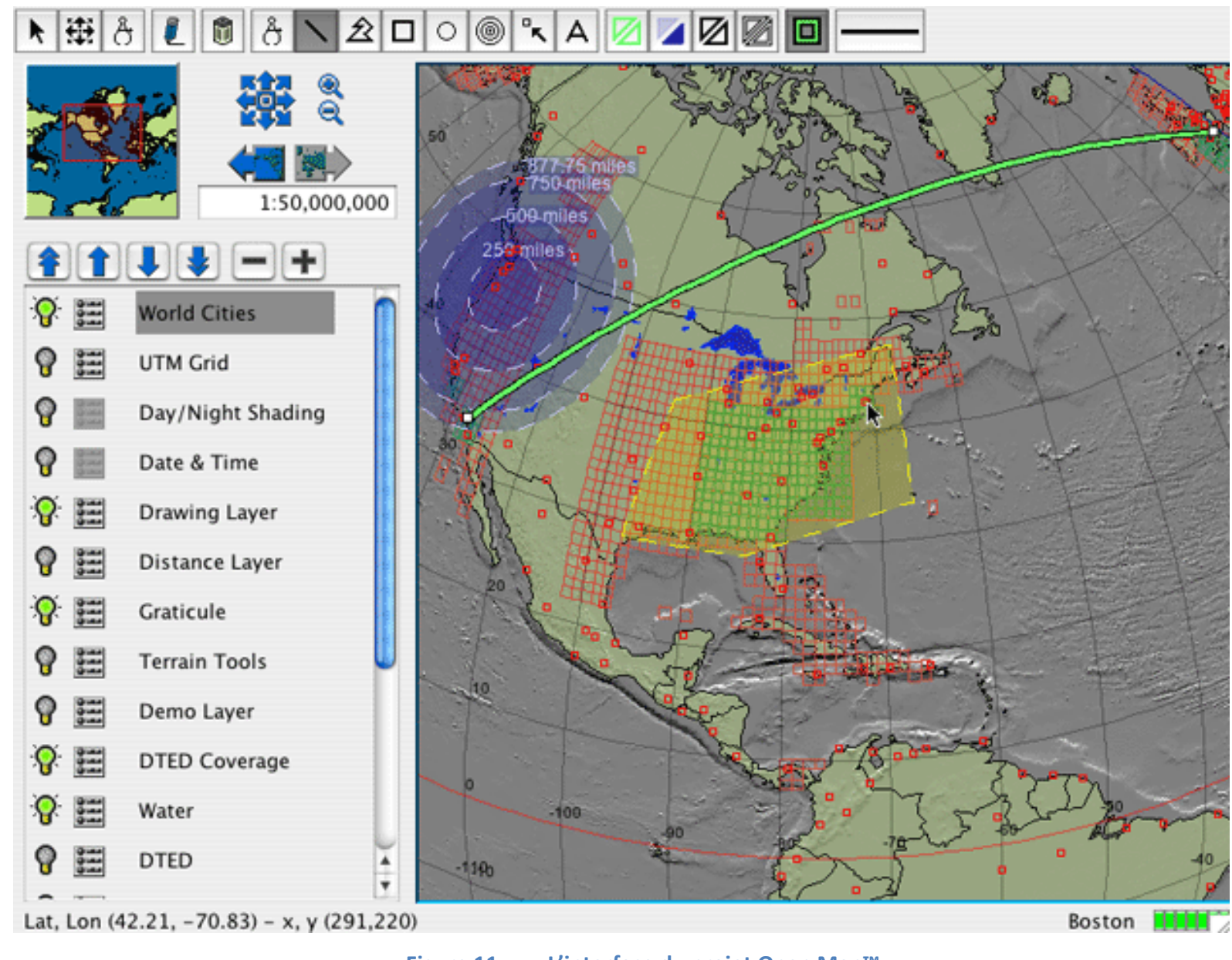

Figure 11. L'interface du projet Open Map ${ }^{\mathrm{TM}}$.

- L'acquisition et la maintenance de données géographiques ont toujours un coût élevé et ces données ne sont pas mises gracieusement à la disposition d'un large public. A part pour certains pays tels les Etats-Unis, où les données sont collectées par des institutions gouvernementales et mises à disposition du public de manière presque gratuite, les géodonnées reviennent donc très chères à l'achat. Ceci est une des principales barrières pour les projets de « web mapping » à budget réduit. Ceci est lié à un autre inconvénient du « web mapping » que constituent les problèmes des droits d'auteurs et de protection de « l'œuvre » cartographique. Ce qui est d'autant plus complexe à gérer compte tenu des législations spécifiques à chaque pays. Le projet INSPIRE a toutefois permis l'harmonisation de certaines de ces questions.

- $\quad$ Enfin, concernant l'affichage de telles cartes sur des terminaux mobiles, se pose le problème de la taille limitée de l'écran. En effet, ces terminaux mobiles ne disposent que d'une résolution d'affichage limitée souvent à maximum 200 x 200 pixels. Les avancées technologiques devraient toutefois combler ces lacunes.

Concernant le domaine du transport, les cartes Internet se retrouvent sur 2 principaux types de sites Internet [12]: 
- Les sites offrant des informations concernant l'état du trafic (derniers incidents, densité de trafic, conditions météorologiques etc.) à travers des cartes, du texte et des images de webcams. Les différentes informations sont recueillies à partir d'un réseau de caméras et capteurs trafic. Les différentes données sont présentées souvent en tant que couches indépendantes d'une même carte de base. C'est l'utilisateur qui choisit quelles couches sont affichées. Les cartes permettant de visualiser ces informations sont soit dynamiques soit interactives. La Figure 12 illustre un exemple d'une telle carte.

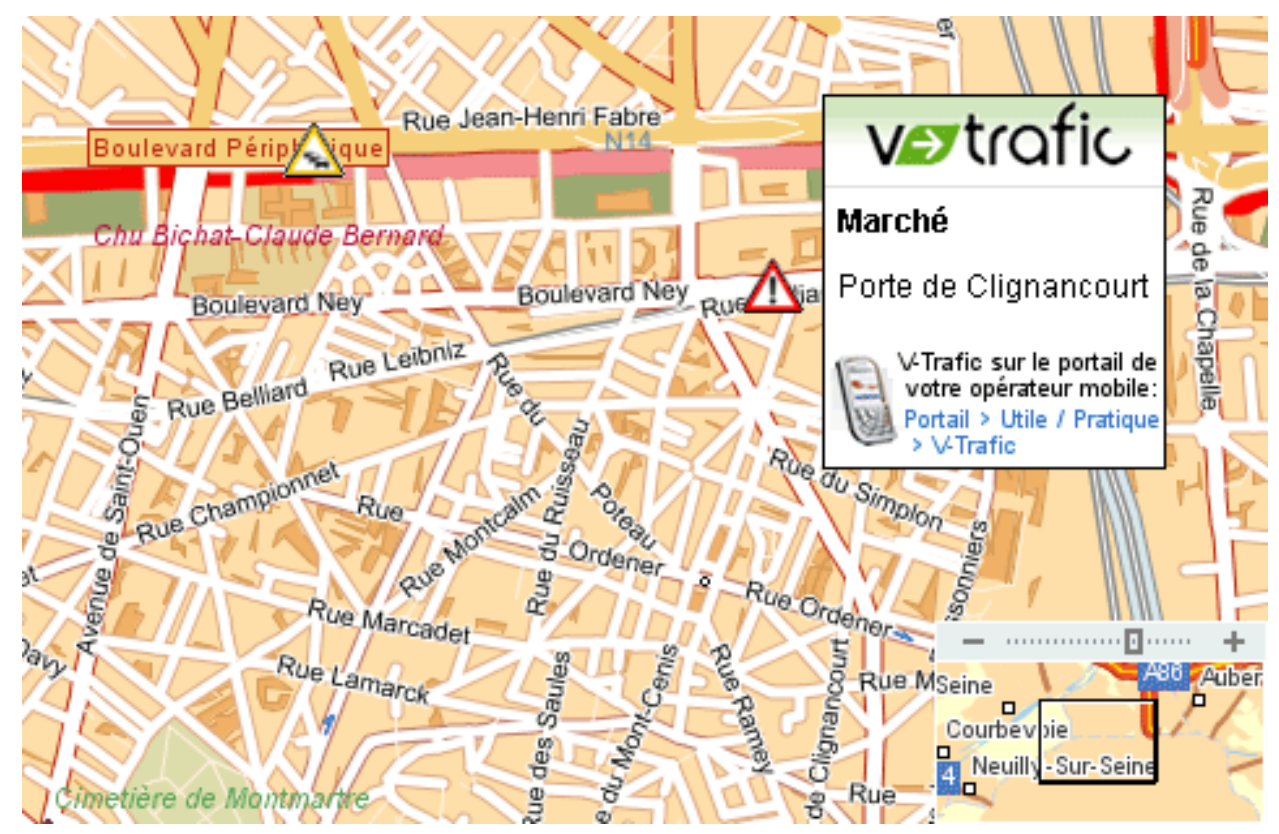

Figure 12. Exemple de carte illustrant l'état du trafic.

(๔ 2008 NAVTEQ, Europa Technologies, Loftmyndir ehf, Basarsoft - Aerodata Intl Surveys)

- Les sites offrant des informations de navigation/guidage prennent de plus en plus en compte l'état du trafic dans leur calcul d'itinéraire. Il s'agit de fournir à l'utilisateur des informations concernant la route en elle-même (type de route, longueur, etc.) et l'itinéraire (durée estimée, distance, etc.). Ces informations sont fournies à travers des cartes accompagnées de texte indiquant les principales étapes de l'itinéraire, notamment les points de départ et d'arrivée [12]. Dans cette catégorie de cartes, nous pouvons aussi citer les cartes permettant la planification de trajets en transports publics. La Figure 13 illustre un exemple d'une telle carte. 


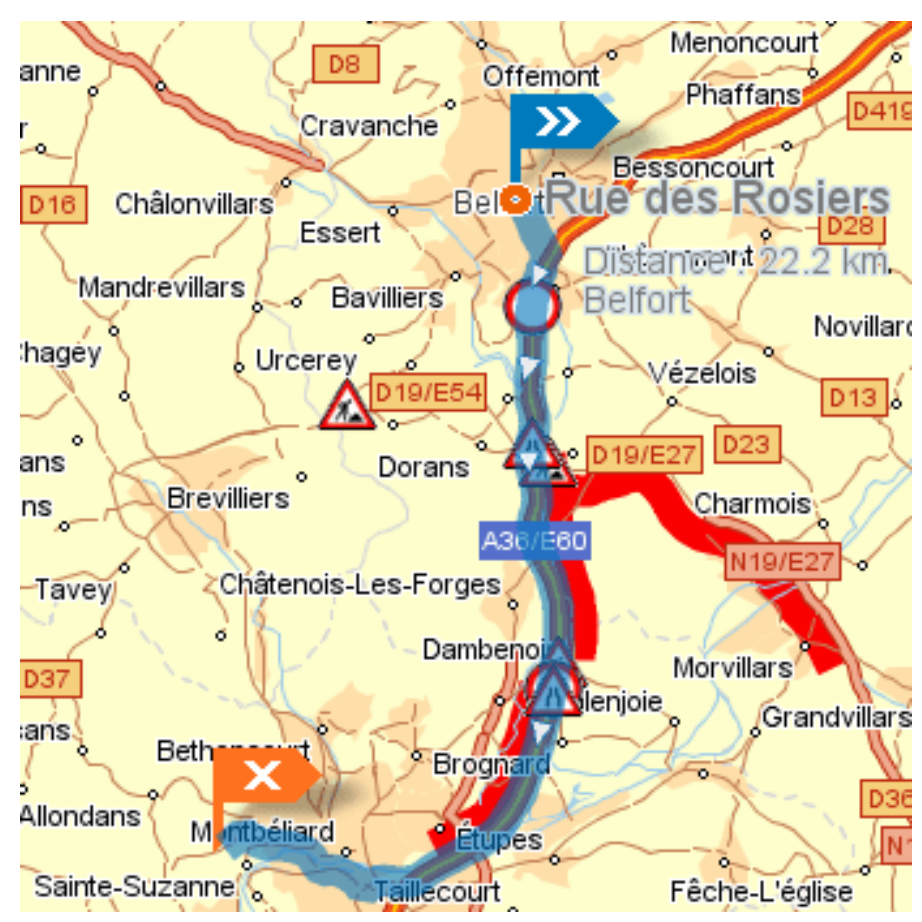

Figure 13. Exemple de carte d'itinéraire intégrant les informations trafic (http://www.fr.map24.com/). (๔ 2008 NAVTEQ, Europa Technologies, Loftmyndir ehf, Basarsoft - Aerodata Intl Surveys)

\section{Modélisation standardisée de l'Information Géographique}

Le but de la modélisation de l'information géographique est de décrire notre environnement, dans un langage que les ordinateurs peuvent comprendre. Les principaux outils informatiques utilisés à cette fin sont les pages Internet et les bases de données. Ces deux outils impliquent des choix de formalismes graphiques, textuels afin de présenter l'information de la meilleure manière possible [13]. Dans les paragraphes suivants, nous allons présenter les principaux langages, standards et formalismes permettant de réaliser ces modélisations d'informations géographiques.

\section{XML (eXtensible Markup Language)}

Le langage XML a été défini par le W3C (World Wide Web Consortium) et il dérive du langage SGML (Standard Generalized Markup Language), défini par le standard ISO 8879.

Le langage XML permet de modéliser les données en utilisant une structure d'arbre. Il s'agit d'une trame permettant de définir des structures de données, sans toutefois décrire comment un document est interprété.

Des règles permettent de définir la structure des documents $X M L$, qui encapsulent les données selon la structure définie. Par contre, pour permettre le traitement de ces documents $X M L$, il faut au préalable que les éléments les composant soient décrits de manière détaillée. Le XML propose deux outils pour réaliser cette description [14]:

- $\quad$ Le DTD (Document Type Definition) est un fichier texte qui contient la « grammaire » ou les « règles de construction » que doivent respecter les documents XML [15].

- $\quad$ Le XML Schema est un fichier XML lui-même, dont le principe est semblable au DTD [15]. Étant un outil plus récent, il permet de pallier à certains défauts du DTD.

Dans le domaine de l'information géographique, le XML est largement utilisé notamment à travers le langage GML, qui sera présenté dans la suite de ce chapitre (voir 3.4.). Pour plus d'informations sur le langage XML, vous pouvez consulter le lien suivant : http://www.w3.org/XML/.

La Figure 14 illustre un exemple de fichier XML représentant une position. 


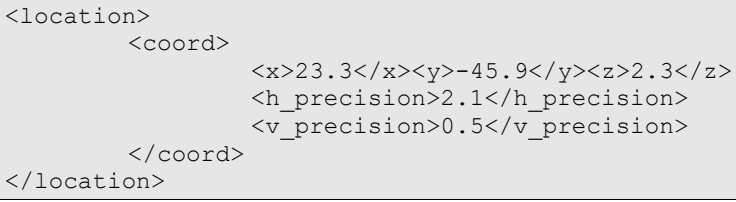

Figure 14. Exemple de fichier XML.

\section{SVG (Scalable Vector Graphics)}

Le SVG est un langage de type « libre de royalties » développé par le W3C. Il s'agit d'un langage basé sur le XML, qui décrit et intègre des graphiques vectoriels, des graphiques rasters, du texte, du multimédia, des l'interactivité, du code et de l'animation [16].

Tel le XML, le langage SVG utilise une structure d'arbre, qui peut être interprétée à la fois par les humains et les ordinateurs. Étant basé sur le XML, le SVG se combine facilement avec d'autres outils de développement modernes, ou encore avec d'autres technologies W3C ou OGC telles : XML, DOM, CSS, JPEG, PNG, SMIL, WMS ou GML [16]. Les interfaces DOM, qui ne dépendent pas d'un langage donné, permettent la modification d'éléments (suppression, création, réarrangement) dans la structure d'arbre du document SVG. Concernant les langages de codage utilisés dans les documents SVG, c'est le JavaScript (ou ECMAScript) qui est le plus utilisé.

Le principal avantage de ce langage est qu'il permet de créer, personnaliser et transformer des objets vectoriels. La qualité du rendu est donc maintenue lorsque l'on augmente ou lorsque l'on diminue l'échelle d'affichage.

Ce standard est utilisé en tant que format d'échange d'objets graphiques. C'est le principal format permettant la création d'applications animées et interactives, notamment pour le « web mapping» [16]. La Figure 15 illustre un exemple d'application « web mapping » utilisant le standard SVG.
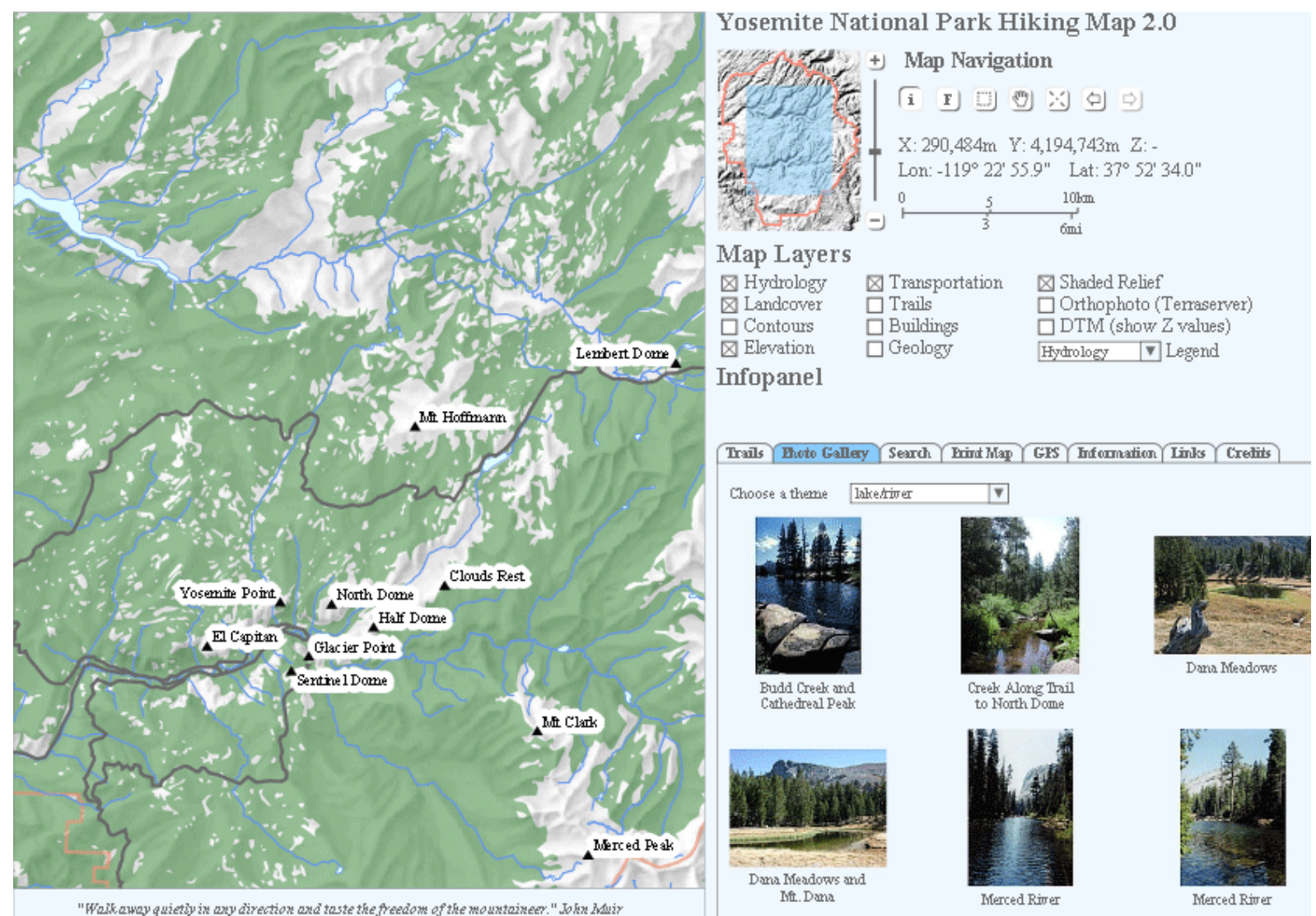

Figure 15. Exemple d'application « web mapping » utilisant le standard SVG (http://www.carto.net/williams/yosemite/) 
Pour plus d'informations concernant ce format d'échange de données, vous pouvez consulter le lien suivant : http://www.w3.org/Graphics/SVG/.

\section{UML (Unified Modeling Language)}

Le langage UML est un langage permettant la spécification de systèmes. Il a été mis au point par plusieurs spécialistes de la modélisation, afin de consolider et standardiser un grand nombre de langages et méthodes de développement apparus au début des années ' 90 dans le domaine des applications orientées-objet [18].

L'UML est en fait une collection de diagrammes permettant de spécifier, modéliser, construire et documenter les systèmes logiciels [17].

L'approche actuelle est de considérer l'UML en tant que standard pour la spécification générale de projets logiciels, notamment dans le développement des SIG et des bases de données spatiales [18]. Afin de répondre au mieux à des problèmes de conception spécifiques à certains domaines, il existe des mécanismes d'extension de l'UML.

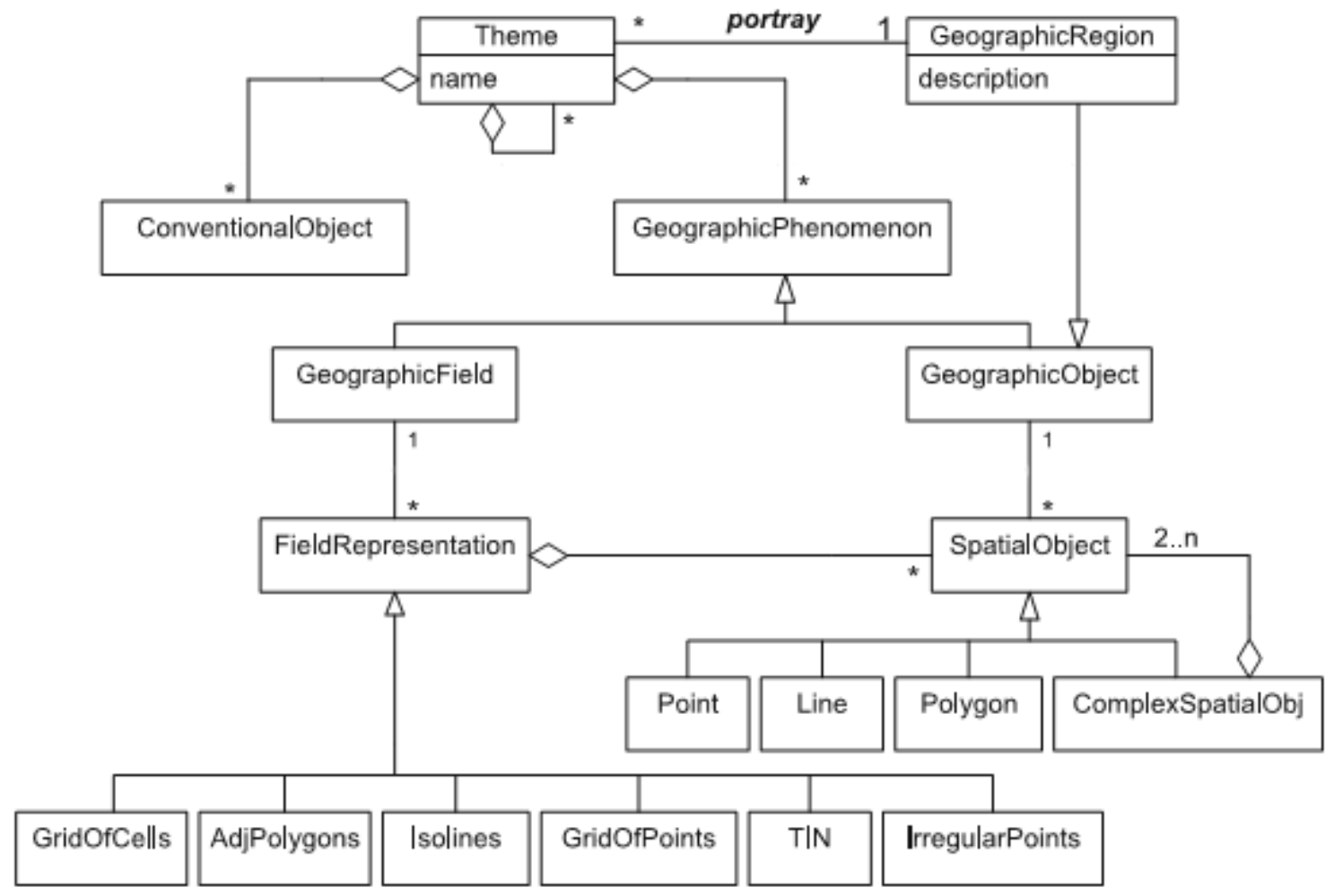

Figure 16. Exemple d’une modélisation UML.

\section{GML (Geography Markup Language)}

Le GML est un langage « open-source » développé par l'OGC, et étant lui aussi basé sur le langage XML [20]. Le GML permet de représenter des objets géographiques. Un objet GML est défini en tant qu'une « abstraction d'un phénomène du monde réel » [19]. Un objet géographique est un objet possédant un attribut de localisation dans un système de référence. Les objets GML sont caractérisés par leurs propriétés et leur état. Chaque propriété est définie en tant qu'une liste de la forme $\{n \circ m$, type, valeur\}. Le langage GML définit les types de propriétés suivants [21]:

- $\quad$ Propriétés géométriques ;

- $\quad$ Propriétés topologiques ;

- $\quad$ Propriétés temporelles ; 
- $\quad$ Propriétés descriptives.

La Figure 17 illustre le diagramme de classes UML du GML :

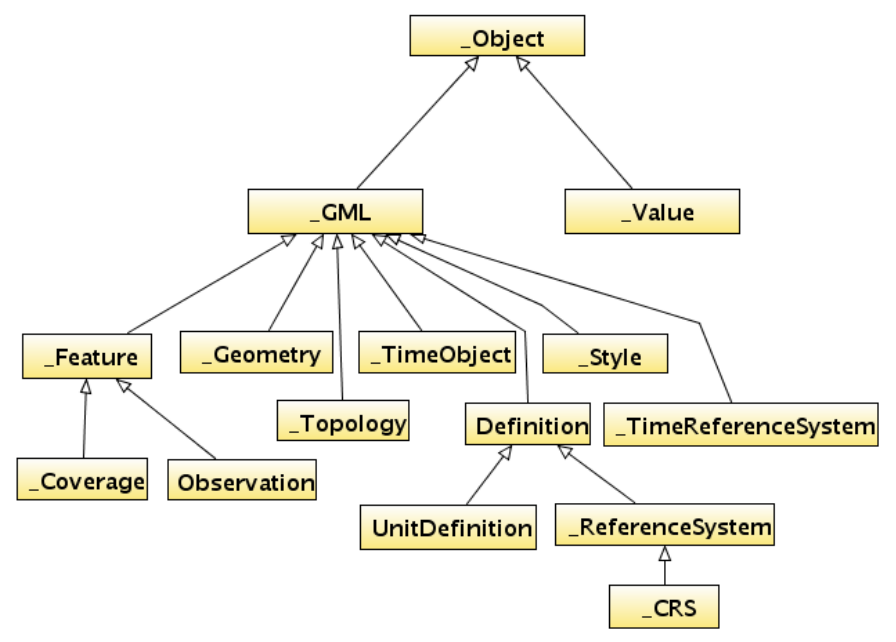

Figure 17. Hiérarchie des classes GML [21].

Le GML a été défini en tant que format d'échange et de stockage d'informations géographiques, permettant leur partage par différents systèmes. Étant largement adoptée par de nombreux acteurs industriels, gouvernementaux ou encore de l'enseignement, la spécification GML a été ajoutée en tant que document de l'ISO. Cependant, il reste encore du travail à faire afin d'améliorer l'usage et l'efficacité du langage GML [20]. Généralement les objets encodés en GML sont beaucoup plus complexes que ceux codés en XML, vu la diversité de propriétés implémentées par le GML. Afin d'assurer une totale compatibilité entre le XML et le GML, les requêtes XML doivent être étendues. De plus, lorsqu'il s'agit d'intégrer le GML pour les équipements mobiles, apparaissent des contraintes de bande passante et capacité de stockage limitée. Toutefois, cette orientation est une des plus prometteuses, dans la mesure où cela permettrait aux terminaux mobiles d'accéder à des sources contenant d'importantes quantités d'informations géographiques.

On peut mentionner ici un langage complémentaire du GML, basé aussi sur un encodage XML, qui est le KML (Keyhole Markup Language) développé par Google. Le KML permet aux utilisateurs de Google Earth d'ajouter leurs propres géodonnées 3D. GML permettant d'encoder des objets géographiques, le KML permet de les afficher ou de les visualiser. Le KML permet donc de transporter des contenus GML. Google a soumis à I'OGC la version 2.2 du langage $\mathrm{KML}$, en vue d'obtenir sa standardisation. Pour une spécification détaillée du standard KML, il faut se reporter à [22].

\section{GDF (Geographic Data Files)}

Dans ses premières versions (dès 1988), le standard GDF a été développé par l'industrie automobile. Le CEN (Comité Européen de Normalisation) TC278 a publié une «pré-norme » en 1995. GDF n'est devenu un standard international qu'en 2004, avec la publication de I'ISO TC204.

Le standard GDF permet la définition et l'échange des données géographiques entre les différents acteurs du domaine de la télématique routière et de la navigation automobile [36]. Ce standard est principalement par les producteurs de cartes numériques pour la navigation automobile, mais aussi par la plupart des services faisant appel à la géolocalisation.

Le standard GDF définit les éléments suivants :

- Un modèle de données indépendant des applications, composé de trois sous-modèles : le modèle des objets décrivant la topologie, le modèle des attributs décrivant le mode de stockage des informations thématiques et le modèle des relations. Ce modèle décrit aussi comment sont représentés les objets et comment le stockage des données est géré. 
- Un dictionnaire de données dépendant des applications, contenant trois catalogues décrivant respectivement les objets, leurs attributs et leurs relations. Parmi les thèmes abordés, on peut citer : les réseaux routiers, les ponts et les tunnels, les chemins de fer, les services et les transports publics, etc.

- Des règles de représentation des objets qui sont décrites pour chaque thème du dictionnaire, et selon chacun des trois niveaux de représentation présentés par la Figure 10.

- $\quad$ Des définitions pour les concepts de qualité et de métadonnées.

- Des spécifications concernant le stockage et le format d'échange des données.

La Figure 18 représente les liens d'agrégation ou de représentation entre les objets d'un niveau et les objets du niveau supérieur. Cette figure n'est pas exhaustive, seuls quelques objets de GDF d'intérêt général sont illustrés.

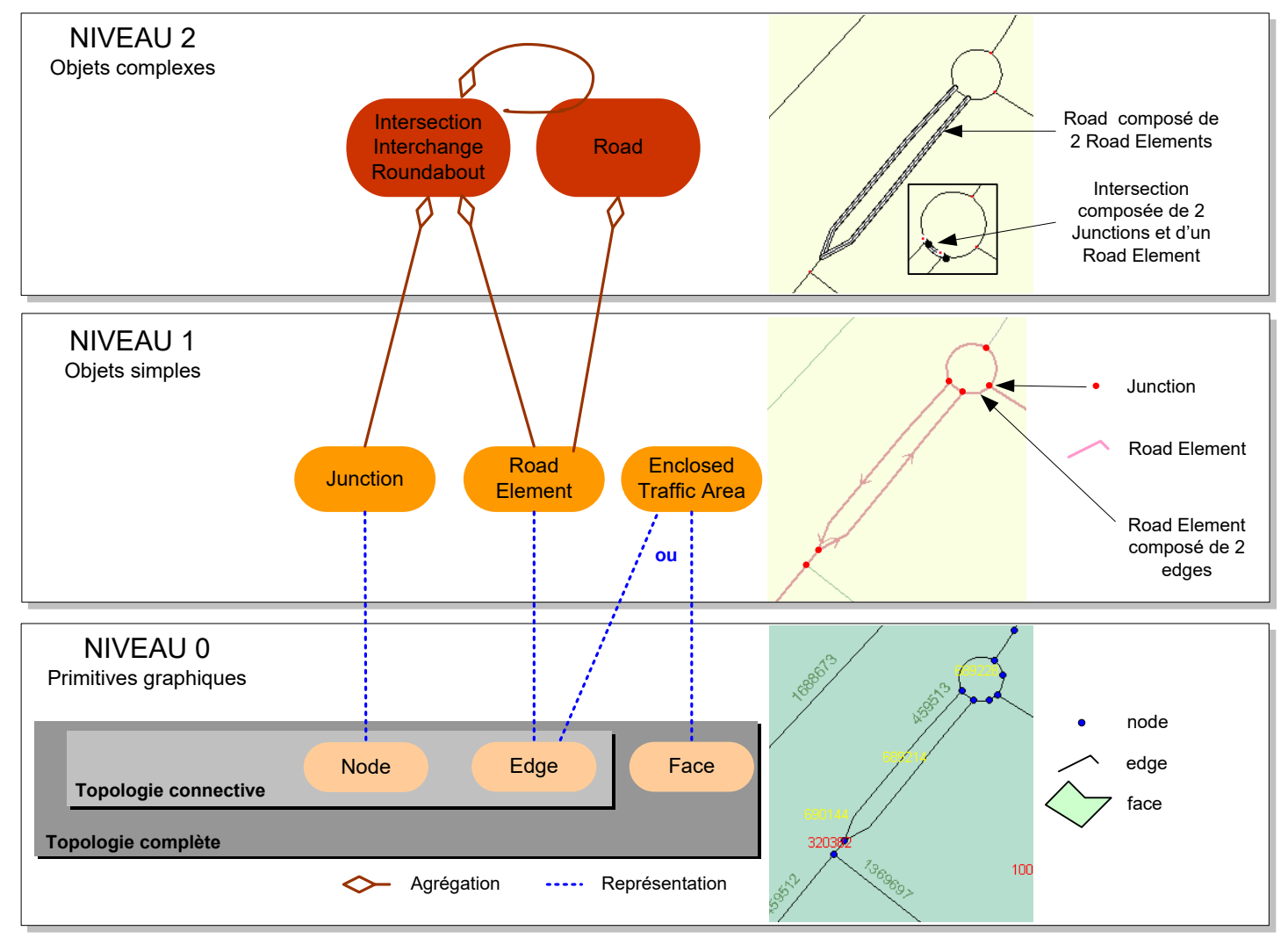

Figure 18. Les trois niveaux de représentation de GDF [36].

\section{Collecte d'Informations Géographiques}

\section{Les méthodes}

\section{Les méthodes photogrammétriques}

La technique de la photogrammétrie exploite une faculté de la vision humaine, à savoir celle de percevoir le relief à partir de deux images planes d'un même objet, mais prises de deux points de vue différents.

La photogrammétrie analogique, classique, est apparue à la fin du 19 ème siècle, en 1859 , et c'est dès la fin des années '60 que l'informatique est utilisée en photogrammétrie avec le développement des restituteurs analytiques. La photogrammétrie numérique est arrivée dans les années ' 80 , avec l'émergence des premiers prototypes de stations de photogrammétrie digitale, dans lesquelles le support du film photographique est remplacé par une image préalablement numérisée. Les premiers systèmes entièrement numériques sont apparus au début des années ' 80 , alors qu'ils ont été commercialisés seulement à partir des années '90 [23]. L'utilisation de caméras aériennes numériques, ainsi que 
l'évolution du matériel photographique, font que, de nos jours, cette technique est de plus en plus utilisée pour la collecte massive d'informations géographiques.

La photogrammétrie numérique est aujourd'hui la solution la plus employée dans le cadre de la cartographie couvrant de larges portions de territoire. Cette méthode permet d'obtenir une modélisation 2D ou 3D d'objets présents dans le monde réel, comme le permet la photogrammétrie classique, mais en utilisant des logiciels de traitement d'image, largement automatisés, fonctionnant sur PC ou autres plates-formes logicielles [24]. Contrairement à la photogrammétrie classique, cette méthode utilise des images saisies numériquement ou des photographies numérisées par scannage à partir de prises de vue stéréoscopiques sur support argentique.

La photogrammétrie numérique utilise donc des images numériques, que l'on peut assimiler à un tableau de pixels, chaque pixel contenant une information couleur. En fonction du codage choisi, sur 8, 16 ou 32 bits, l'information couleur s'étale sur une palette donnée. Par exemple, pour une image codée sur 8 bits, les couleurs seront codées avec des valeurs entre 0 et $255\left(=2^{8}\right)$.

\section{Les méthodes liées au "laser scanning"}

Le « laser scanning » est une technique permettant l'acquisition rapide et précise de surfaces en tant qu'ensembles de points présents sur ces surfaces, notamment appelés «nuages de points ». Ces opérations sont effectuées à partir de plateformes aériennes ou terrestres [25]. Ces techniques complémentaires à la photogrammétrie ont été développées dès les années '90 et se sont imposées comme moyen rapide et précis pour la saisie de modèles numériques de terrain et d'objets dès les années 2000.

- Lorsque le «laser scanning » est effectué à partir de plateformes aériennes, il s'agit de levés laser aéroportés. Les plateformes aériennes sont en fait des avions ou hélicoptères équipés de récepteurs-émetteurs LASER, notamment appelés LiDAR (Light Detection And Ranging) [26]. Les LiDAR balayent la surface à scanner avec des faisceaux laser. Lorsque ces faisceaux sont réfléchis par la surface terrestre ou d'autres objets (bâtiments, forêts, etc.), ces faisceaux sont à nouveau captés par le LiDAR. Le temps de retour de ces faisceaux permet d'estimer la distance entre la surface à saisir et le LiDAR. La précision altimétrique ainsi atteinte est de l'ordre du décimètre. Pour ce faire, la position et I'orientation du LiDAR doit être connue avec précision. Cette position est obtenue grâce à un système GPS ainsi qu'une centrale inertielle. Une station GPS au sol permet d'augmenter la précision du positionnement afin de traiter les mesures en mode différentiel.

\section{[à insérer] \\ Figure 19. Principe du LiDAR aéroporté.}

- $\quad$ Les levés laser terrestres, autrement appelés LiDAR terrestres, sont effectués à partir de stations de scannage montées sur un trépied. L'instrument mesure simultanément les angles verticaux et horizontaux du faisceau laser qui lui fournit la distance à l'objet. Le principe de la mesure électronique des distances par laser se fait soit par la mesure du temps de vol d'un signal (aller et retour) ou par une mesure de déphasage de l'onde porteuse.

\section{Figure 20. Principe du LiDAR terrestre.}

De nos jours, les levés du type LiDAR se diversifient et sont utilisés dans un nombre grandissant d'applications, autant dans la recherche, dans l'industrie que dans les sociétés de services (géomatiques, géophysiques, etc.). Parmi les applications de télédétection LiDAR, on peut citer la création de modèles numériques d'élévation, les modèles urbains $3 \mathrm{D}$, la bathymétrie fluviale et océanique ou encore l'étude de la surface terrestre [27]. Ces applications seront présentées plus en détail dans le la section "Sources thématiques". 


\section{Les produits}

\section{Les produits de la photogrammétrie}

On définit un «produit de la photogrammétrie » comme étant une représentation de certains aspects d'une scène (objet ou terrain), dérivés à partir d'une image de la scène [28]. Cette représentation peut être géométrique, topologique et inclure des coordonnées de points, des géométries d'objets ou encore des mesures. [29] présente un état de l'art complet sur les différentes techniques, méthodes et applications de la photogrammétrie. Ce document contient aussi de nombreuses illustrations de produits de la photogrammétrie.

\section{Les produits maillés}

Afin de pouvoir exploiter les images brutes résultant d'un processus de photogrammétrie, il est d'abord nécessaire de relier la position des objets présents dans l'image à leur position dans le monde réel [28]. Les images brutes sont d'abord traitées afin d'obtenir des images plus facilement exploitables. Parmi les images traitées, on recense les catégories suivantes [28]:

- Les images "géo-rectifiées » sont produites en projetant l'image brute sur une surface de référence. Les images numériques ainsi traitées sont projetées soit sur un plan de référence, soit sur une surface ellipsoïde, comme c'est le cas pour les images satellitaires. Ce traitement permet de supprimer l'effet de perspective, mais ne corrige pas les décalages provoqués par les différentes altitudes présentes dans l'image [28]. Une image satellitaire représente de nombreux objets géographiques, mais leurs attributs ne sont pas connus directement. Contrairement à la carte, il n'existe pas de légende pour indiquer les attributs de ces objets géographiques. Les images satellitaires sont généralement utilisées en combinaison avec d'autres données géospatiales, telles des cartes ou des graphiques numériques. Ces images, de la même manière que les ortho-photographies, permettent d'identifier des modifications de la surface terrestre (aménagements du territoire, couverture forestière, cours des rivières, etc.). Les images satellitaires peuvent être consultées de plusieurs manières différentes :

- A partir de sites Internet, tels Géoportail de I'IGN (http://www.geoportail.fr/), ou encore TerraServer (http://www.terraserver.com/),
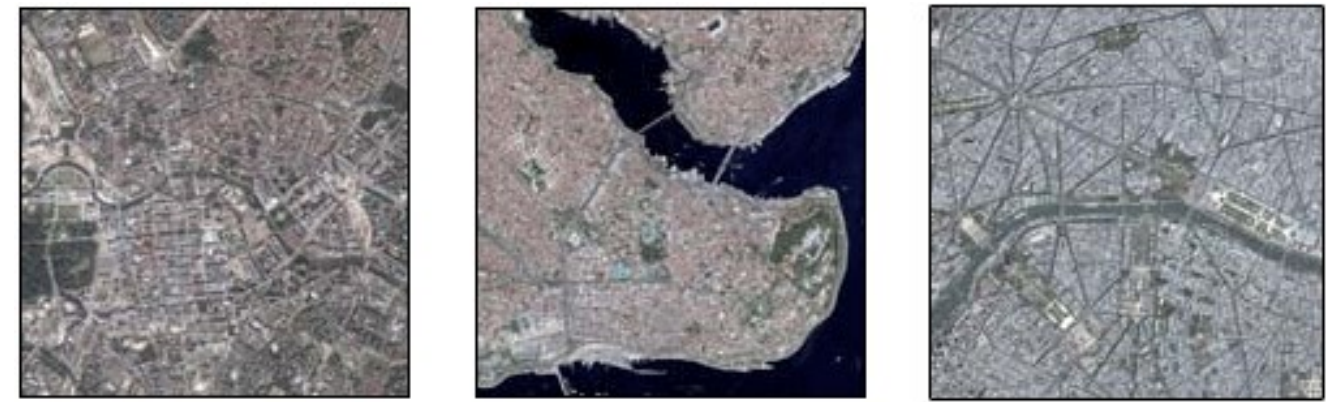

Figure 21. Exemples d'images géo-rectifiées, telles qu'affichées sur TerraServer

(Copyright @ 2007 - TerraServer $^{\circledR}$ )

- A partir de logiciels d'imagerie satellitaire ou «globes virtuels », tels Google Earth (http://earth.google.fr/), NASA World Wind (http://worldwind.arc.nasa.gov/) ou encore Microsoft Virtual Earth (http://www.microsoft.com/VirtualEarth/). Ces logiciels permettent de superposer aux images satellitaires des calques d'informations, comme par exemple les noms de rues ou les noms des villes.

- Les images "ortho-rectifiées» (ou encore les ortho-photographies) sont construites en réalisant une projection orthographique de l'image brute. Dans le cas d'une telle projection, la direction de projection est perpendiculaire au plan cartographique [28]. Une ortho-photographie est une image calculée en fonction de la position et de l'orientation de la caméra au moment de la prise de vue, mais aussi en fonction du relief. Le relief est déterminé à travers des modèles numériques de terrain. La Figure 22 en illustre les principales étapes [24, 30, 31]. 


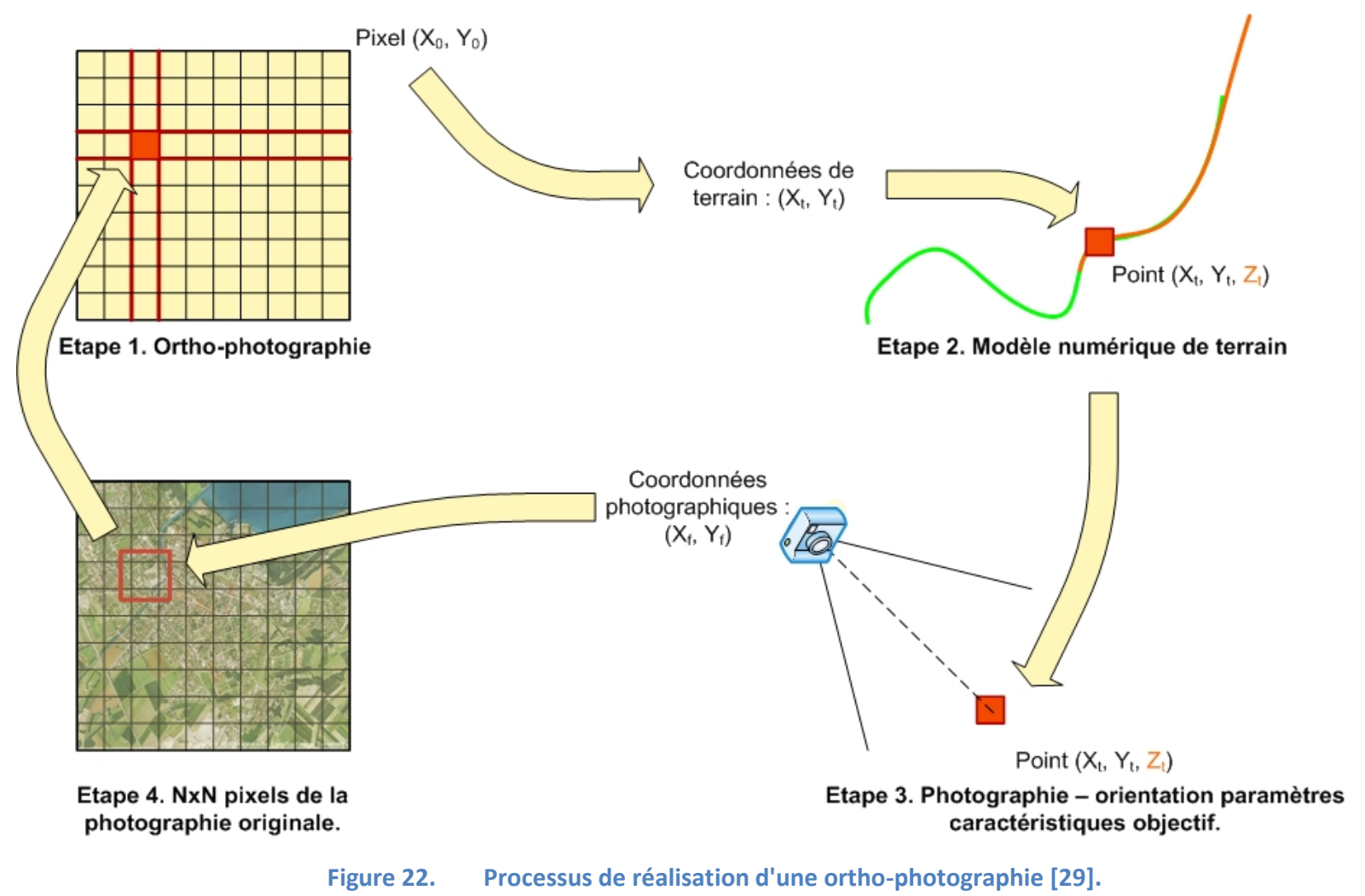

\section{Les produits vectoriels}

Les produits vectoriels sont en fait des représentations des objets ou des zones présentes dans la scène ou la portion de territoire considérée. Ces représentations vectorielles sont le plus souvent utilisées pour décrire des routes, des immeubles, des zones naturelles ou construites. Les représentations vectorielles englobent trois représentations [28]:

- La représentation géométrique - La géométrie d'un objet vectoriel peut contenir soit les coordonnées de points individuels, soit une forme géométrique prédéfinie, telle un arc de cercle, un rectangle, etc. Les éléments d'une représentation géométrique sont des points, des lignes ou des polygones [28]. S'il s'agit de représentations $2 D$, seules les coordonnées $X$ et $Y$ sont considérées. Lorsqu'une troisième coordonnée, l'altitude $Z$, est intégrée, on parle de représentation « $2,5 \mathrm{D}$ », car les faces non- horizontales des objets 3D (tels les immeubles) ne sont pas représentées de manière explicite. Une vraie représentation 3D contient des informations concernant les faces des objets, notamment la direction de la normale à leur surface.

- La représentation topologique - Les éléments d'une représentation topologique sont des points, des arêtes et des faces [28]. Les nœuds sont des liens entre 2 ou plusieurs arêtes, contrairement aux points qui ne font qu'indiquer une position. Une arête est formée par un ensemble ordonné de points. Une arête a un côté gauche et un côté droit, et peut délimiter une face. Une représentation topologique permet de raisonner sur les propriétés d'une scène donnée. Ce type de représentation permet des raisonnements concernant les relations spatiales entre les objets, telles que « à côté de... », « adjacent à... » ou encore « connecté à... ».

- $\quad L a$ représentation sémantique - Ce procédé permet de stocker les propriétés des différents objets vectoriels [28]. Dans le cas d'une représentation vectorielle d'une route, des objets vectoriels seront utilisés pour indiquer le type de surface (asphalte, graviers, etc.), le nombre de voies de circulation ou encore le type de route. Dans le cas d'immeubles, il s'agira surtout de définir leur usage (résidentiel, commercial, industriel, etc.) ou le type de construction (briques, bois, etc.).

\section{Modèles d'altitudes}

Les modèles d'altitude représentent l'élévation de la surface de la Terre. On distingue deux types de modèles : 
- Le modèle numérique de terrain (MNT) - ensemble de points décrivant la surface au sol,

- Le modèle numérique de surface (MNS) - ensemble de points bruts décrivant tous les objets (sol, constructions, forêts, etc.).

Pour créer un MNT, on utilise des mesures photogrammétriques de points et de lignes, obtenues soit à partir de courbes de niveau numérisées, soit à partir de levés sur le terrain, soit à l'aide de mesures laser effectuées à partir d'un avion.

Un MNT est un modèle de représentation de l'altitude dans un fichier maillé. Le but est de représenter un ensemble de points ayant chacun des coordonnées 3D dans un système de référence.

Le but d'un MNT est de donner une description du relief pour une zone géographique déterminée. C'est pour cette raison que les MNT sont aussi appelés modèles numériques d'élévation. Un MNT peut correspondre à des terres émergées, des fonds sous-marins ou des fonds de rivières [32].

En fonction de l'application finale, deux types de représentations sont utilisés [28]:

- Les représentations maillées, ou grilles raster, sont les plus utilisées, puisque très faciles à afficher et à manipuler. Pour chaque point, seule la coordonnée « $z$ » (l'altitude) est enregistrée. Un exemple d'une telle représentation est donné par la Figure 23.

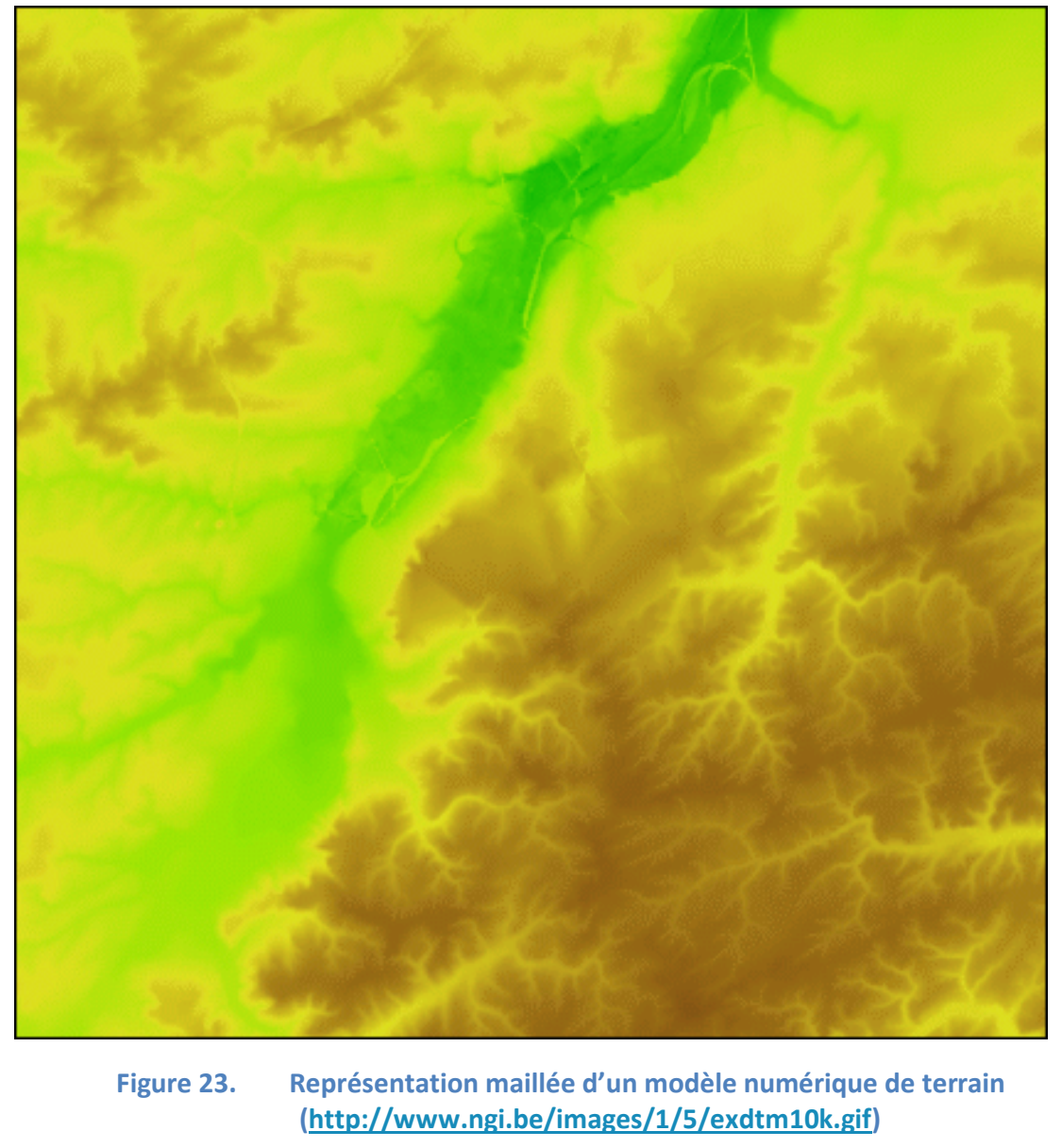

- Les représentations en réseau de triangles ou TIN (Triangulated Irregular Network) sont formés d'un ensemble de points distribués de manière irrégulière, connectés 2 par 2 afin de former une surface de triangles connexes. Les représentations TIN sont plus efficaces que les représentations raster, notamment en termes d'espace de stockage nécessaire pour une qualité ou une précision équivalentes. Même si les représentations TIN nécessitent 3 coordonnées $(x, y, z)$ pour chaque point, le gain d'espace de stockage se fait en jouant sur la concentration de points pour une zone donnée [28]. En effet, sur les zones complexes, plusieurs points sont concentrés, alors que sur des zones plus homogènes la densité de points est beaucoup plus faible. Un exemple d'une telle représentation est donné par la Figure 24. 


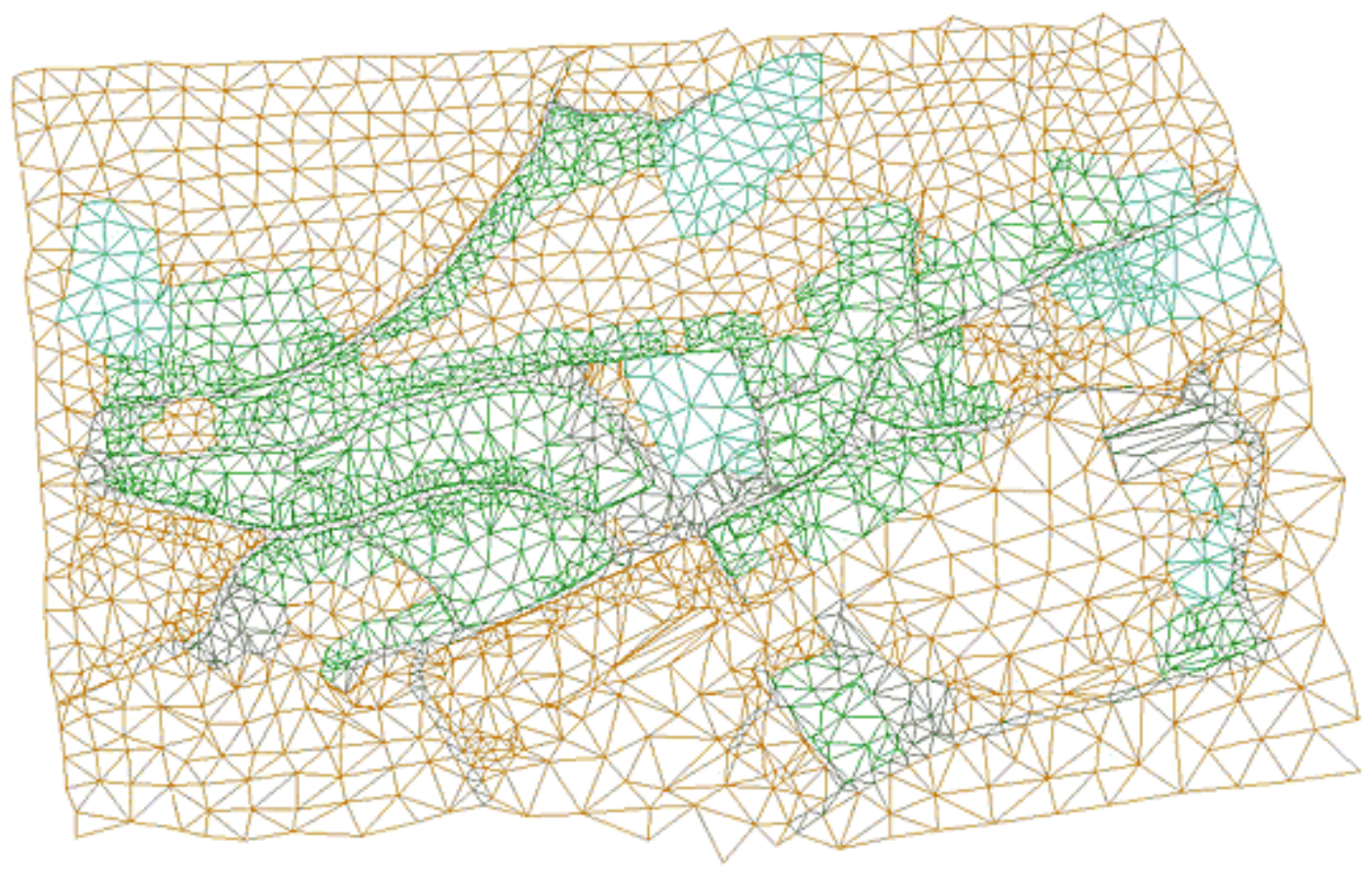

Figure 24. Modèle numérique de terrain - modèle TIN (http://www.ngi.be/images/2/2/8/tinmodel.gif)

- Les représentations de type courbes de niveau ont longtemps été le standard pour la représentation des MNT et restent le moyen universel de représenter l'altimétrie sur une carte. Aujourd'hui, ces représentations peuvent être facilement dérivées à partir des représentations raster ou TIN. Un exemple d'une telle représentation est donné par la Figure 25.

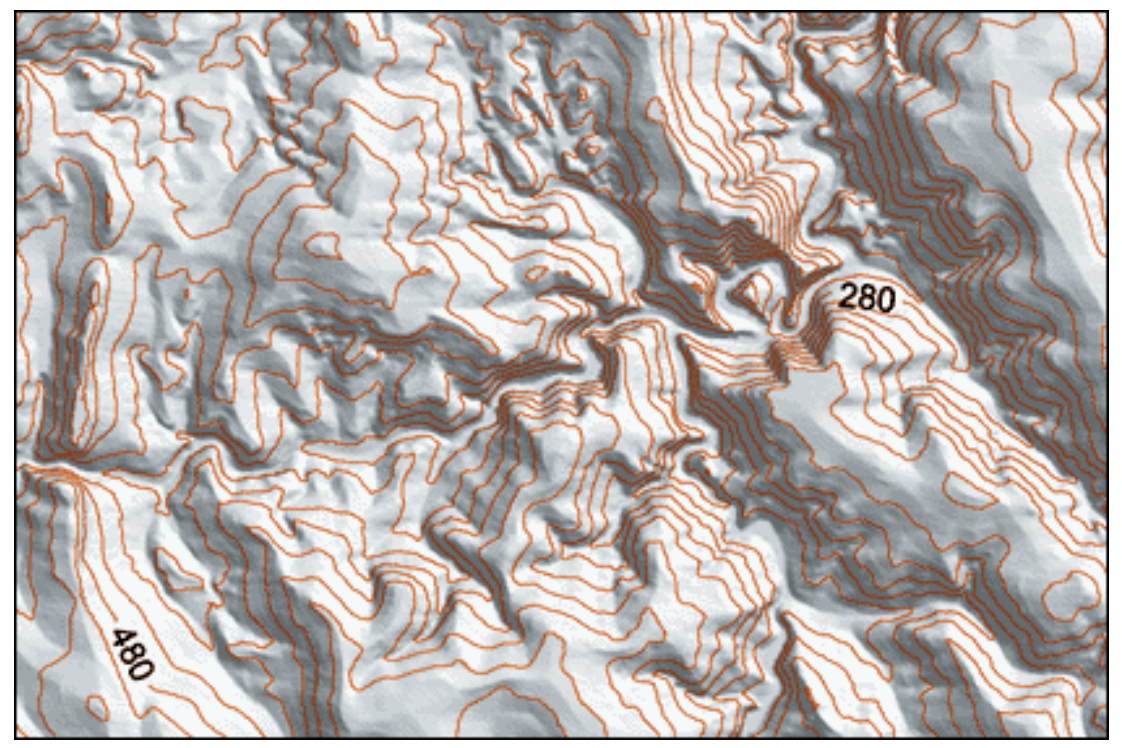

Figure 25. Exemple de modèle numérique d'élévation (http://www.ngi.be/images/1/5/extdted50d.gif)

Modèles urbains 3D et modèles du paysage

Les modèles urbains 3D et les modèles du paysage sont parmi les produits les plus répandus de la photogrammétrie et de l'imagerie, utilisés à la fois par des applications de visualisation ou SIG. La plupart des modèles photogrammétriques 3D sont collectés en tant qu'éléments vectoriels [28]; il s'agit de modèles dans lesquels seuls les sommets et les arêtes de l'objet sont représentés. Les faces des objets ainsi modélisés ne sont pas connues, les objets sont donc transparents. Cette représentation des 
objets 3D a l'avantage de permettre la combinaison aisée de plusieurs de ces objets vectoriels de manière à former des objets $3 \mathrm{D}$ plus complexes.

Souvent, les modèles complets, donc incorporant les informations des faces, appliquent des textures à ces faces afin d'avoir une apparence plus réelle. C'est en effet la tendance actuelle concernant les modèles urbains 3D, dont l'aspect visuel devient de plus en plus important [33]. Un exemple d'un tel modèle est donné par la Figure 26.

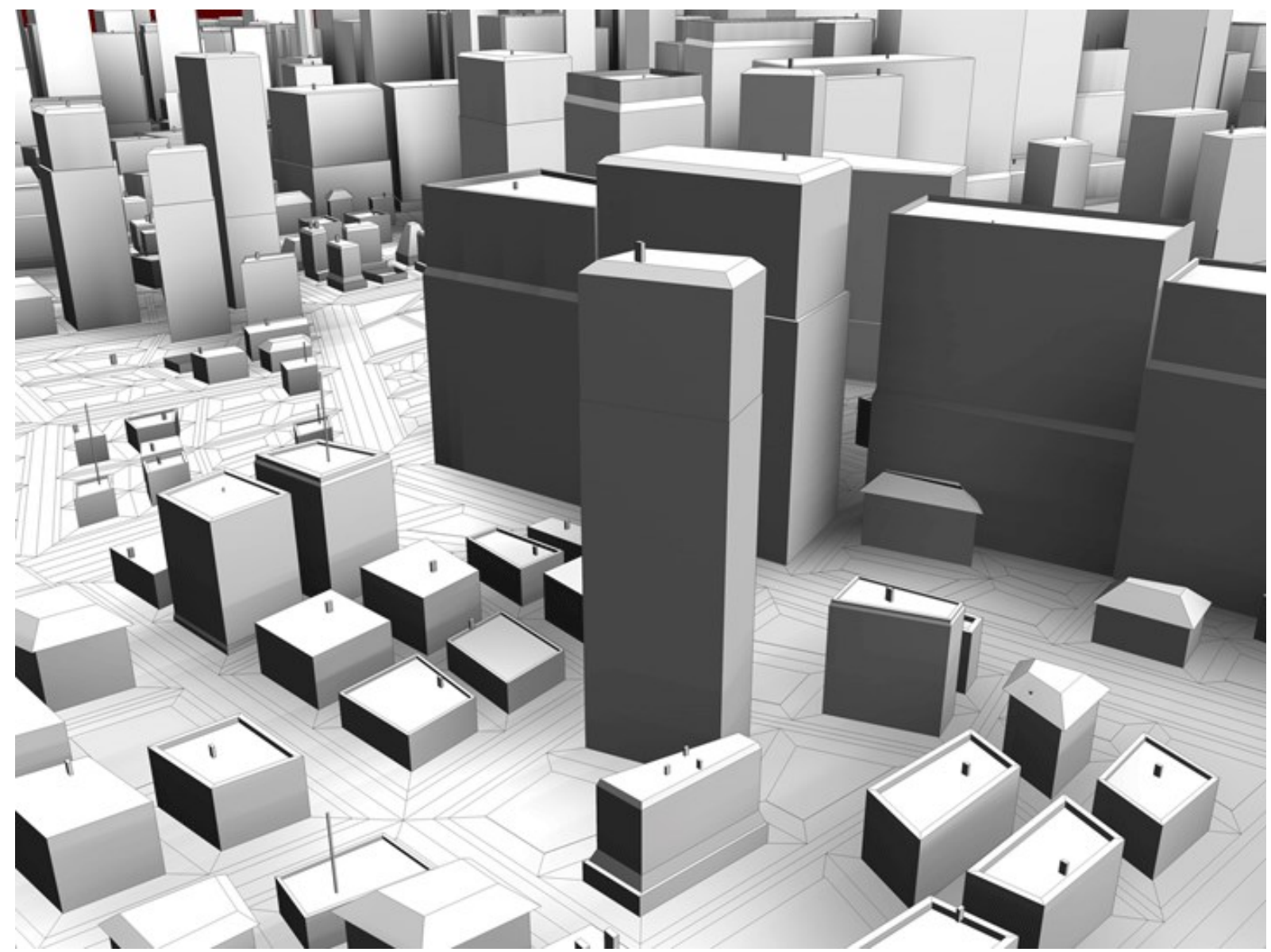

Figure 26. Exemple de modèle urbain 3D.

Les produits du «laser scanning»

Le développement des techniques de LiDAR aéroportées a été grandement motivé par la nécessité d'acquérir des modèles numériques des régions forestières. Au fur et à mesure, les techniques de LiDAR aéroportées sont devenues les techniques préférées pour l'acquisition de modèles numériques pour tout type de terrain [25]. Les nuages de points ainsi récoltés contiennent de nombreuses informations qui sont extraites en vue d'autres applications, telles la cartographie à grande échelle de zones limitrophes à des routes, des rails ou des lignes électriques.

\section{[à insérer]}

Figure 27. Image produite par un LiDAR aéroporté.

Concernant les techniques de LiDAR terrestre, celles-ci trouvent des applications principalement dans le domaine des installations industrielles, de la reconstitution de scènes ou encore en vue de préserver le patrimoine culturel. Le principal avantage de cette technique est qu'elle permet une grande densité de points allant jusqu'à 1 point $/ \mathrm{cm}^{2}$ pour des applications à courte distance [25]. C'est la raison pour laquelle, les levés LASER terrestres permettent de créer des modèles très détaillés de façades d'immeubles/bâtiments, intégrés dans des modèles urbains 3D à grande échelle. 


\section{Sources d'Informations Géographiques}

\section{Métadonnées et catalogues de données}

Le terme «métadonnées » désigne un ensemble de données renseignant sur la nature et les caractéristiques d'autres données [34]. Dans le domaine de l'information géographique, des métadonnées géographiques sont utilisées. Elles permettent une utilisation judicieuse et pertinente des données géographiques. Les métadonnées géographiques permettent d'identifier les données géographiques disponibles, leurs utilisations, leur qualité, comment les trouver ou comment y accéder.

Concernant la standardisation des métadonnées, au niveau international, c'est la norme ISO 19115 du comité technique ISO/TC 211 «Information géographique / Géomatique », qui a été adoptée au mois de mai 2003. Il s'agit d'une norme générale, qui s'applique à tout type de données géographiques [34]. Le texte complet de la norme peut être trouvé sur le site de l'ISO/TC $211:$ http://www.isotc211.org. Cette norme s'inspire en partie de la norme CSDGM (Content Standard for Digital Geospatial Metadata), mise au point par le FGDC (Federal Geographic Data Commitee) aux Etats-Unis. Le profil français de la norme EN-ISO 19115 est disponible à partir du site du CNIG (Conseil National de I'Information Géographique) : http://www.cnig.gouv.fr/.

L'OGC a, lui aussi, publié des recommandations et des normes concernant la spécification des métadonnées dans les SIG [35]. Celles-ci peuvent être trouvées parmi les spécifications du groupe de travail « Metadata Working Group», sur le site de l'OGC : http://www.opengeospatial.org.

Parmi les autres standards pour les métadonnées, on peut citer l'initiative du DGIWG (Digital Geographic Information Working Group), qui a publié en 1994 le standard DGIEST (Digital Geographic Information Exchange Standard) [35], auquel il est possible d'accéder à partir de l'adresse suivante : https://www.dgiwg.org/digest/.

Le MIT (Massachusetts Institute of Technology) a publié une liste de liens vers les différentes normes de métadonnées existantes. L'accès à cette liste se fait à travers le site de la bibliothèque du MIT [35]: http://libraries.mit.edu/guides/subjects/metadata/standards.html.

\section{Sources d'Informations Géographiques}

Aujourd'hui l'information géographique est très riche et variée, non seulement de par sa nature et ses origines, mais aussi de par sa précision et sa validité géographique.

Les sources d'informations géographiques sont innombrables et souvent il faut faire preuve d'ingéniosité et d'énergie pour aller dénicher l'information recherchée. C'est la source d'une information géographique, en principe décrite par des métadonnées, qui en définit la précision, sa validité sémantique, spatiale ou temporelle. Une information « brute » sera donc plus intéressante que sa mise en forme agrégée, puisqu'elle sera plus détaillée et qu'elle pourra être mieux interprétée en fonction de l'objectif recherché.

Les sources d'information géographique peuvent être classées en deux groupes principaux :

- les sources de données géographiques de référence,

- les sources de données géographiques thématiques.

Ces données peuvent provenir soit de sources d'informations classiques qui ont été numérisées, soit de nouvelles sources (images satellitaires, photos aériennes).

Pour accéder aux données numériques, il faut en général s'adresser directement à l'organisme producteur ou à ses diffuseurs. Une liste des principaux producteurs en France est disponible sur le site 
du CNIG. La mise à disposition des données est généralement assortie d'une licence précisant les conditions d'utilisation et de reproduction.

\section{Sources de référence}

L'essentiel de ces sources est apporté par les données diffusées soit par l'IGN pour la topographie terrestre, soit par le cadastre pour les limites de propriété foncière, soit par le SHOM (Service Hydrographique et Océanographique de la Marine) pour le domaine maritime. Le tableau suivant permet d'identifier quelques unes sources d'informations géographiques de référence.

\begin{tabular}{|c|c|}
\hline $\begin{array}{l}\text { Source de données } \\
\text { géographiques }\end{array}$ & Description \\
\hline IGN & 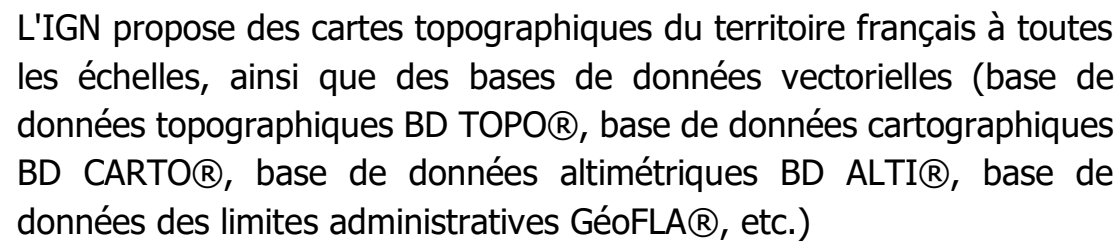 \\
\hline Cadastre & $\begin{array}{l}\text { Le plan cadastral représente le contour de toutes les parcelles foncières } \\
\text { présentes sur un territoire donné, avec l'ensemble du bâti et de la voirie, } \\
\text { ainsi que l'indication de l'adresse postale de chaque parcelle. }\end{array}$ \\
\hline SHOM & $\begin{array}{l}\text { L'information hydrographique fournit le repérage de base nécessaire à } \\
\text { l'ensemble des activités maritimes, en particulier en matière de } \\
\text { navigation, où le problème de la sécurité constitue une priorité } \\
\text { essentielle. Parmi les différents types de données fournis, on peut citer } \\
\text { les bases de données cartographiques marines, les données } \\
\text { bathymétriques générales de la Zone d'Extension Française (ZEE), qui } \\
\text { représente } 11 \text { millions de } \mathrm{km}^{2} \text {, ou encore les balises maritimes et feux, } \\
\text { les câbles sous-marins, les épaves et obstructions, ainsi que les zones } \\
\text { maritimes règlementées (séparation de trafic, zones interdites) figurant } \\
\text { dans les zones de souveraineté française. }\end{array}$ \\
\hline
\end{tabular}

Tableau 2. Sources de référence pour les données géographiques.

\section{Sources thématiques}

Ces sources sont à la fois très nombreuses et très diverses. Elles peuvent porter sur certaines sciences de la Terre (géologie, météorologie, etc.), ou sciences humaines (démographie, économie) mais aussi sur certains domaines d'activités (transports, agriculture, etc.). Le panorama ci-dessous est forcément incomplet. Le tableau suivant permet d'identifier quelques sources d'informations géographiques thématiques.

\begin{tabular}{|l|lllll|}
\hline $\begin{array}{l}\text { Type des données } \\
\text { géographiques }\end{array}$ & Sources \\
\hline $\begin{array}{l}\text { Données géologiques et } \\
\text { géophysiques }\end{array}$ & $\begin{array}{l}\text { Bureau de Recherches Géologiques et Minières } \\
\text { http://www.brgm.fr/ }\end{array}$ \\
\hline $\begin{array}{l}\text { Données concernant le } \\
\text { sol, l'agriculture, la forêt }\end{array}$ & $\begin{array}{l}\text { Institut National de la Recherche Agronomique } \\
\text { http://www.inra.fr/ } \\
\text { Inventaire Forestier National (IFN) }-\underline{h t t p: / / w w w . i f n . f r / ~}\end{array}$ & - \\
\hline
\end{tabular}




\begin{tabular}{|c|c|}
\hline & $\begin{array}{l}\text { Centre de recherche pour l'ingénierie de l'agriculture et de } \\
\text { l'environnement (CEMAGREF) - } \text { http://www.cemagref.fr/ }\end{array}$ \\
\hline $\begin{array}{l}\text { Données concernant } \\
\text { l'environnement }\end{array}$ & Institut Français de l'ENvironnement (IFEN) - http://www.ifen.fr/ \\
\hline $\begin{array}{l}\text { Données concernant la } \\
\text { météo }\end{array}$ & Météo France - http://www.meteo.fr/ \\
\hline $\begin{array}{l}\text { Données concernant la } \\
\text { population et l'activité } \\
\text { économique }\end{array}$ & $\begin{array}{l}\text { Institut National de la Statistique et des Etudes Economiques (INSEE) - } \\
\underline{\text { http://www.insee.fr/ }}\end{array}$ \\
\hline $\begin{array}{l}\text { Données concernant les } \\
\text { infrastructures }\end{array}$ & $\begin{array}{l}\text { Ministère de l'Equipement, des Transports et du Logement - } \\
\text { http://www.equipement.gouv.fr/ }\end{array}$ \\
\hline Données territoriales & $\begin{array}{l}\text { Ces sont des données collectées et gérées par les services techniques } \\
\text { des collectivités territoriales (Conseils régionaux, généraux, communes), } \\
\text { et qui concernent les mêmes domaines d'activités listés plus haut, mais } \\
\text { au plan local et à grande échelle. }\end{array}$ \\
\hline Données trafic & $\begin{array}{l}\text { InfoTrafic - http://www.infotrafic.com/home.php } \\
\text { V-Trafic - http://www.v-trafic.com/ }\end{array}$ \\
\hline
\end{tabular}

Tableau 3. Sources thématiques de données géographiques.

\section{Conclusions}

En guise de conclusion, le recensement intégral de l'ensemble des sources de données géographiques est une tâche impossible à réaliser. Grâce au développement des technologies modernes, autant pour la communication avec Internet, que pour le positionnement avec les systèmes satellitaires, tels le GPS, GLONASS et à l'avenir Galileo, les informations géographiques se sont grandement diversifiées. Les sources d'informations géographiques se sont, elles aussi, multipliées. La Figure 29 est une vue d'ensemble des représentations géographiques. Cette figure présente une classification des différentes formes de représentations des objets géographiques. Toutes ces représentations ont été présentées et illustrées dans ce chapitre. 


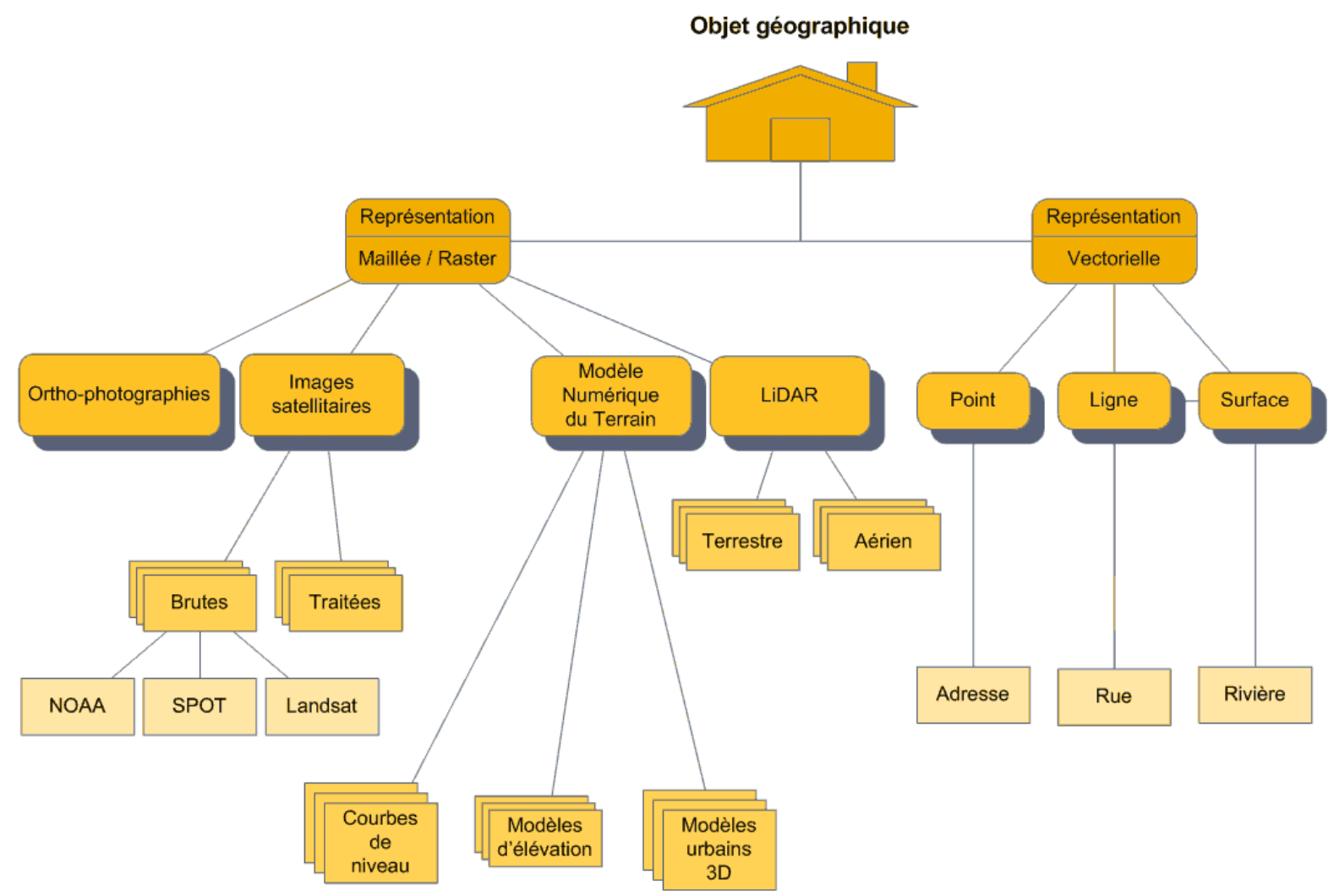

Figure 29. Classification des formes de représentation des objets géographiques.

Pour conclure, l'information géographique, de sources variées, ainsi que de différents types, est aujourd'hui de plus en plus exploitée. De par le passé, le problème était de trouver l'information géographique. De nos jours, vu l'abondance des sources d'information géographique, un nouveau problème se pose. Il s'agit en effet de la qualité de l'information géographique, autrement dit de la précision de la description du monde réel, intrinsèque aux données géographiques. Il s'agira dorénavant de choisir la « bonne » représentation et non plus la « bonne » information.

\section{Références}

[1] Souris M., 2002, La construction d'un système d'information géographique, principes et algorithmes du système SAVANE, Thèse de Doctorat, Université de La Rochelle, $505 \mathrm{p}$.

[2] Lim, H., Raster Data, In : Shekhar, Xiong (eds.) Encyclopedia of GIS, Page(s): 949-955, Springer Science, 2008

[3] V. Gandhi, Vector Data, In : Shekhar, Xiong (eds.) Encyclopedia of GIS, Page(s): 1217-1221, Springer Science, 2008

[4] http://www.statcan.ca/francais/freepub/12-591-XIF/2006001/glossary f.htm (visité le 21/11/2008)

[5] Golay, F., Cours Géoinformation - Introduction: qu'est-ce qu'une carte et quelques questions d'échelle, LaSIG, EPFL, disponible en ligne à l'adresse suivante: http://lasig.epfl.ch/enseignement/cours/geoinfo1/Geoinfo Cours1_Cartographie.pdf (visité le 21/11/2008)

[6] Caloz, R., Collet, C., Précis de télédétection - Volume 3, Presses de I'Université du Québec, 2001.

[7] A. Roxin, Gaber, J., Wack, M., Location based models for pervasive road networks, IEEE International Conference on Pervasive Services (ICPS), Page(s):443 - 447, 15-20 July 2007, disponible en ligne à partir de l'adresse suivante : 
http://ieeexplore.ieee.org/iel5/4283874/4283875/04283954.pdf?arnumber=4283954 (visité le 21/11/2008)

[8] George, B. ; Shekhar, S., Digital Road Maps, In: Shekhar, Xiong (eds.) Encyclopedia of GIS, p. 967-972, Springer Science, 2008

[9] Kraak, M.-J., Settings and needs for web cartography, In: Kraak, Brown, A (eds.) Web Cartography. p. 3-4, Taylor \& Francis, New York (2001)

[10] Ormeling, F., Atlas information systems, In: Proceedings of the 17th International Cartographic Conference of the ICA, Barcelona, vol. 2, p. 2127-2133 (1995)

[11] Siekierska, E.M. ; Taylor, F.D.R., Electronic Mapping and Electronic Atlases: New Cartographic Products for the Information Era - The Electronic Atlas of Canada, CSIM Journal ACSGC, 45(1), 11-21 (1991)

[12] http://www.kartografie.nl/webcartography/webbook/ch12/ch12.htm (visité le 21/11/2008)

[13] http://lasig.epfl.ch/projets/modelisation/Manuel/concepts.shtml (visité le 21/11/2008)

[14] Lupp, M., Extensible Markup Language, In : Shekhar, Xiong (eds.) Encyclopedia of GIS, Page(s): 307, Springer Science, 2008

[15] http://lasig.epfl.ch/projets/modelisation/Manuel/XML.shtml (visité le 21/11/2008)

[16] Neumann, A., Scalable Vector Graphics (SVG), In : Shekhar, Xiong (eds.) Encyclopedia of GIS, Page(s): 1013-1021, Springer Science, 2008

[17] http://lasig.epfl.ch/projets/modelisation/Manuel/UML.shtml (visité le 21/11/2008)

[18] Filho, J. L. ; Braga, J. L., UML : Unified Modeling Language, In : Shekhar, Xiong (eds.) Encyclopedia of GIS, Page(s): 1183-1184, Springer Science, 2008

[19] http://lasig.epfl.ch/projets/modelisation/Manuel/GML.shtml (visité le 21/11/2008)

[20] Raimundo, F. ; Dos Santos, J.R. ; Chang-Tien L., Geography Markup Language (GML), In : Shekhar, Xiong (eds.) Encyclopedia of GIS, Page(s): 364-368, Springer Science, 2008

[21] http://www.opengis.net/gml/ (visité le 21/11/2008)

[22] http://www.opengeospatial.org/standards/kml/ (visité le 21/11/2008)

[23] Hellwich, O., Photogrammetric Methods, In : Shekhar, Xiong (eds.) Encyclopedia of GIS, Page(s): 860-864, Springer Science, 2008

[24] Henry, J.B., Cours de Topographie et Topométrie Générale, Chapitre 4 - Les méthodes indirectes, Service Régional de Traitement d'Image et de Télédétection, disponible en ligne à I'adresse suivante : http://jb.henry.free.fr/cours/chapitre 4.pdf (visité le 21/11/2008)

[25] Vosselman, G., Laser Scanning, In : Shekhar, Xiong (eds.) Encyclopedia of GIS, Page(s): 609- 612, Springer Science, 2008

[26] De Joinville, O. ; Saur, S. ; Bretar, F., Le levé laser aéroporté : techniques, applications et recherche, Bulletin d'information scientifique et technique de I'IGN, numéro 74, 2003/3, disponible en ligne à l'adresse : http://recherche.ign.fr/doc/BI74/B03 74 JOINVILLE.pdf (visité le $21 / 11 / 2008$ )

[27] Cuesta, J., Les applications Lidar aux études physiques et environnementales, Institut Pierre Simon Laplace / Laboratoire de Météorologie Dynamique, disponible en ligne à l'adresse suivante : www.sfp2007grenoble.fr/latex/resumes/MLB3426/MLB3426.pdf $\quad$ (visité le 21/11/2008)

[28] McGlone, J. C., Photogrammetric Products, In : Shekhar, Xiong (eds.) Encyclopedia of GIS, Page(s): 864-871, Springer Science, 2008

[29] La photogrammétrie à I'IGN, article disponible en ligne à l'adresse suivante: http://www.ngi.be/Common/articles/PT/photogrammetrie.pdf (visité le 21/11/2008) 
[30] La Photogrammétrie aérienne, article disponible en ligne à l'adresse suivante: http://www.ign.fr/telechargement/P.I/IGNMagazine/Reportage IGNMag7.pdf(visité 21/11/2008)

[31] http://www.swisstopo.admin.ch/internet/swisstopo/fr/home/products/images/ortho.ht ml (visité le 21/11/2008)

[32] http://fr.wikipedia.org/wiki/Mod\%C3\%A8le num\%C3\%A9rique de terrain (visité le 21/11/2008)

[33] Paparoditis, N. ; Bentrah, O. ; Deveau, M. ; Tournaire, O. ; Pénard, L., Modélisation 3D automatique terrestre d'environnements urbains et complexes à très grande échelle, Bulletin d'information scientifique et technique de I'IGN, numéro 75, 2006/1, disponible en ligne à l'adresse : http://recherche.ign.fr/doc/BI75/B02 75 PAPARODITIS.pdf (visité le 21/11/2008)

[34] ISO 19115-1, Information géographique - Métadonnées - Partie 1: Principes de base

[35] Semerjian, C. J., Metadata, In : Shekhar, Xiong (eds.) Encyclopedia of GIS, Page(s): 652-655, Springer Science, 2008

[36] ISO 14825:2004, Systèmes intelligents de transport (SIT) - Fichiers de données géographiques (GDF) - Spécification des données globales 
